The ASTROPHYSICAL JOURNAL SUPPLEMENT SERIES, 122:415-430, 1999 June

(C) 1999. American Astonomical Society. All rights reserved. Printed in U.S.A.

\title{
SURVEY FOR EMISSION-LINE GALAXIES: UNIVERSIDAD COMPLUTENSE DE MADRID LIST $3^{1}$
}

\author{
O. Alonso, ${ }^{2}$ C. E. García-Dabó, J. Zamorano, J. Gallego, and M. Rego \\ Departament Astrofísica, Universidad Complutense de Madrid, E-28040 Madrid, Spain; oal@eucmos.sim.ucm.es \\ Received 1998 July 1 ; accepted 1999 January 19
}

\begin{abstract}
A new low-dispersion objective-prism search for low-redshift $(z<0.045)$ emission-line galaxies (ELGs) has been carried out by the Universidad Complutense de Madrid using the Schmidt Telescope at the Calar-Alto Observatory. This is a continuation of the UCM Survey, which was performed by visual selection of candidates in photographic plates via the presence of the $\mathrm{H} \alpha+[\mathrm{N}$ II $] \lambda 6584$ blend in emission. In making this new list we have applied an automatic procedure, fully developed by us, for selecting and analyzing ELG candidates on the digitized images obtained with the Machine Automatique à Mesurer pour l'Astronomie (MAMA). The analyzed region of the sky covers $189 \mathrm{deg}^{2}$ in nine fields near $\alpha=14^{\mathrm{h}}$ and $17^{\mathrm{h}}, \delta=25^{\circ}$. The final sample contains 113 candidates. Special effort has been made to obtain a large amount of information directly from our uncalibrated plates by using several external calibrations. The parameters obtained for the ELG candidates allow us to study the statistical properties of the sample.

Subject headings: galaxies: Seyfert — surveys - techniques: image processing
\end{abstract}

\section{INTRODUCTION}

The Universidad Complutense de Madrid Survey (UCM Survey) has been carried out during the last several years with the aim of looking for $\mathrm{H} \alpha$ emission-line galaxies. It is described in detail by Zamorano et al. $(1994,1996)$ in UCM Lists 1 and 2.

The UCM Survey was initiated with several objectives, the main goals being (1) to identify and study new young, low-metallicity galaxies, (2) to carry out the classification and determination of the overall properties and completeness of the sample of emission-line galaxies (ELGs) selected, and (3) to determine the spatial distribution and luminosity function of the new galaxy population. We also wished to compare our survey with others and to discover the differences between the samples obtained with various objectiveprism techniques, and to study the overall relation between the far-infrared properties and the optical behavior of the star-forming galaxies. Finally, we intended to determine the evolutionary status and the different underlying stellar populations of the objects, in order to detect any effect of evolution in the starburst phenomena and to quantify the properties of star formation in the local universe.

The final product of Lists 1 and 2 was the Universidad Complutense de Madrid (UCM) sample of star-forming galaxies in the local universe. This sample has been observed and analyzed in detail (by Vitores et al. 1996a, 1996b using optical imaging; by Gallego et al. 1996, 1997 using spectroscopy; and by Alonso-Herrero et al. 1996 and Gil de Paz et al. 1998 using near-infrared imaging), and has provided the luminosity function for the star-forming galaxies and the density of the star formation rate in the local universe (Gallego et al. 1995).

\footnotetext{
${ }^{1}$ Based on observations collected at the German-Spanish Astronomical Center, Calar Alto, Spain, operated jointly by the Max-Planck Institut für Astronomie (MPIA), Heidelberg, and the Spanish National Commission for Astronomy.

${ }^{2}$ Present address: Departament Arquitectura de Computadores y Automática, Universidad Complutense de Madrid, E-28040 Madrid, Spain.
}

Lists 1 and 2 suffer from the subjective technique of looking for candidates by visual inspection of the plates through a $10 \times$ binocular microscope. We intend to overcome this drawback by automatic selection on digitized plates. We have shown that this method provides good performance and an improvement in the selection of candidates by applying it to several fields (Alonso et al. 1995; Alonso 1996). In this work we present the UCM List 3 obtained using the automatic detection process in nine contiguous fields covering $189 \mathrm{deg}^{2}$.

Section 2 describes the instrumental setup and observations. In $\S 3$ the method used to select the candidates is outlined. Section 4 presents several parameters measured for every galaxy of the sample and explains how they were obtained. In $\S 5$ the known selection effects are discussed. Finally, some statistical properties and a comparison with other surveys are presented in $\S \S 6$ and 7, respectively.

\section{OBSERVATIONAL DATA}

This survey is based on photographic plates obtained with the $80 / 120 \mathrm{~cm} \mathrm{f} / 3 \mathrm{Schmidt}$ Telescope of the Calar Alto German-Spanish Observatory (Almería, Spain) (Birkle 1984) in 1993 June. All the fields acquired for this research were obtained using the direct and objective-prism configurations. In both modes, we use $8 \times 10$ inch $^{2}$ plates, covering a useful field of $4.4 \times 5.5 \mathrm{deg}^{2}$, with a plate scale of $86^{\prime \prime}$ $\mathrm{mm}^{-1}$. The plates were hypersensitized by baking them in an atmosphere of forming gas for $2 \mathrm{hr}$ prior to exposure. Full details of the plates are listed in Table 1.

Direct plates were obtained by exposing IIIaJ plates, combined with the GG385 filter, for $1 \mathrm{hr}$. This configuration gives an instrumental response similar to the Johnson $B$ band. Prism plates were acquired in the red region of the spectrum. The use of the IIIaF emulsion, which has a sharp red cutoff at $6850 \AA$, and a RG630 filter provides a useful spectral range from $\sim 6400$ to $6850 \AA$. The objective-prism plates were obtained through a full-aperture $4^{\circ}$ prism that yields a dispersion of $1980 \AA \mathrm{mm}^{-1}$ at $\mathrm{H} \alpha$ (see $\left.\S 4.3\right)$. Dispersion of the prism spectra runs along the north-south axis. Our experience with the instrumental setup used indicates 
TABLE 1

Plate Data

\begin{tabular}{|c|c|c|c|c|c|c|}
\hline \multirow{2}{*}{$\begin{array}{l}\text { Plate IDENTIFICATION: } \\
\text { OP/DIRECT } \\
(1)\end{array}$} & \multicolumn{2}{|c|}{ Plate Center (J2000) } & \multirow{2}{*}{$\begin{array}{l}\text { COMMON } \\
\text { FIELD } \\
\text { (deg) } \\
(4)\end{array}$} & \multirow{2}{*}{$\begin{array}{c}\text { SEEING: } \\
\text { OP/DIRECT } \\
(\operatorname{arcsec}) \\
(5)\end{array}$} & \multicolumn{2}{|c|}{ NUMBER OF } \\
\hline & $\begin{array}{l}\text { R.A. } \\
\text { (2) }\end{array}$ & $\begin{array}{l}\text { Decl. } \\
\text { (3) }\end{array}$ & & & $\begin{array}{c}\text { Objects } \\
\text { (6) }\end{array}$ & $\begin{array}{c}\text { ELGs } \\
(7)\end{array}$ \\
\hline A516/A503 & 144656 & +235927 & $4.15 \times 5.14$ & $1.0 / 1.5$ & 14213 & 13 \\
\hline A513/A510 & 142915 & +235345 & $4.24 \times 5.20$ & $2.5 / 1.0$ & 14932 & 14 \\
\hline A495/A507 ............ & 141312 & +234925 & $3.92 \times 5.04$ & $1.5 / 1.0$ & 16999 & 9 \\
\hline A497/A506 ............ & 135638 & +235739 & $4.06 \times 5.13$ & $2.0 / 1.0$ & 17321 & 25 \\
\hline A496/A505 ............. & 174052 & +242334 & $4.15 \times 4.92$ & $1.5 / 2.0$ & 85601 & 19 \\
\hline A498/A509 ............ & 172317 & +243357 & $4.15 \times 5.05$ & $2.0 / 1.0$ & 75618 & 13 \\
\hline A500/A504 ............ & 170555 & +243009 & $4.07 \times 5.15$ & $1.0 / 1.0$ & 47559 & 10 \\
\hline A514/A508 ........... & 165001 & +240437 & $4.13 \times 5.17$ & $2.5 / 1.0$ & 27558 & 5 \\
\hline A517/A512 ........... & 163224 & +240430 & $4.07 \times 5.13$ & $1.0 / 1.0$ & 29583 & 5 \\
\hline
\end{tabular}

NoTE.-Units of right ascension are hours, minutes, and seconds, and units of declination are degrees, arcminutes, and arcseconds.

that exposures $2 \mathrm{hr}$ long are a good balance between depth and plate background. This instrumental configuration registers the $\mathrm{H} \alpha+[\mathrm{N} \mathrm{II}]^{3}$ blend in emission for objects up to $z \approx 0.045$.

Quality standards for the analyzed plates are good seeing, no cloud interference, no exposure interruption, and telescope near the meridian. Excellent guiding was achieved on this observing run through the use of the new automatic guiding system installed on the Schmidt Telescope.

The plates were scanned using the Machine Automatique à Mesurer pour l'Astronomie (MAMA $)^{4}$, a highperformance multichannel microdensitometer located at the Observatoire de Paris (Guibert \& Moreau 1991; Moreau 1992). We used a pixel size and sampling step of 10 $\mu \mathrm{m}$, the highest resolution achieved by the machine. Each scanned plate returns a $23 \mathrm{~K} \times 18 \mathrm{~K}$ pixel image at 0 "' 86 pixel $^{-1}$.
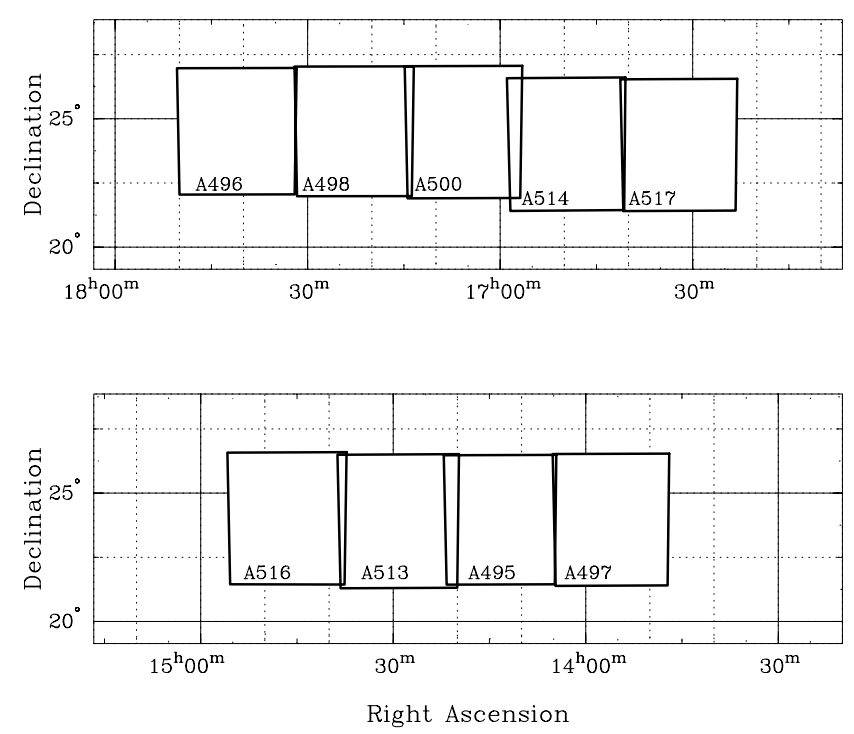

FIG. 1.- Schematic map of the sky, showing the field covered by the UCM List 3. Field centers are provided in Table 1.

\footnotetext{
${ }^{3}$ The combined $\mathrm{H} \alpha+[\mathrm{N} \mathrm{II}]$ emission will be referred to in the following discussion simply as the $\mathrm{H} \alpha$ emission.

${ }^{4}$ MAMA (Machine Automatique à Mesurer pour l'Astronomie) was developed and is operated by CNRS/INSU (Institut National des Sciences de l'Univers) and is located at the Observatoire de Paris.
}

Figure 1 shows the fields covered by this discovery list. They are placed in two regions located in the areas $13^{\mathrm{h}} 45^{\mathrm{m}}<\alpha<14^{\mathrm{h}} 55^{\mathrm{m}}, 21^{\circ}<\delta<27^{\circ}$ and $16^{\mathrm{h}} 25^{\mathrm{m}}<\alpha<$ $17^{\mathrm{h}} 50^{\mathrm{m}}, 21^{\circ}<\delta<27^{\circ}$. The total surveyed area is $189 \mathrm{deg}^{2}$.

\section{DATA REDUCTION AND SELECTION OF CANDIDATES}

In order to decrease the uncertainties intrinsic to visual scanning, we have developed a new method for the automatic selection of emission-line candidates in digitized objective-prism plates. The full procedure is described in detail in Alonso et al. (1995) and Alonso (1996). Here we present a brief discussion of the method.

The first task must be to find all the objects on the plates. In order to locate and measure objects in the astronomical images, we have developed a program that thresholds the image a certain number of $\sigma$ over the local sky background. It returns a catalog with several parameters, such as positions, sizes, fluxes, etc., for all the identified objects with a minimum selectable size. Our BUHOFITS software is able to handle large FITS files, up to our $23 \mathrm{~K} \times 18 \mathrm{~K}$ pixel images. The large number of spurious identifications due to emulsion flaws, plate scratches, or satellite tracks are rejected by cross-correlating the catalogs obtained from direct and prism plates for the same field. We have used a plot of the logarithm of the area versus density flux to discriminate between stellar-like and extended objects (Reid \& Gilmore 1982).

Subsequently, the spectra of all the objects are extracted by adding the central five scans (i.e., those with higher signal) from the objective-prism image. These monodimensional spectra are obtained in arbitrary and instrumental density units. No calibration spots were recorded on the plates to obtain a photographic density-to-intensity relation. Nevertheless, the emission feature is clearly registered in these uncalibrated spectra and will be used in all the subsequent analyses. As a result of the spectrum extraction procedure, it should be noted that we are missing those galaxies with emission out of the central region. This is one of our selection effects, but it only affects very bright, extended, and well-known galaxies.

The emission-line galaxy candidates are selected by analyzing the prism spectra for all the objects. We use three different criteria to perform this selection, as described in detail in Alonso et al. (1995). In our low-dispersion and uncalibrated plates, all the spectra show a similar appear- 
ance, mainly dominated by the instrumental response. Only objects showing the $\mathrm{H} \alpha$ line in emission will present a clear discrepancy with respect to the continuum spectra of stars and galaxies without emission. The selection criteria are based in this fact, and allow us to compute a selection index for each object that informs us about the reliability of the presence of an emission feature. The final sample is obtained after visual inspection of those objects with the largest selection indexes. This step is needed because several different configurations, such as overlapping stars, emulsion scratches in the prism image, etc., can produce extracted spectra with an apparent strong emission feature.

The UCM survey had a typical success rate of $70 \%$ when looking for ELGs (Lists 1 and 2). The remaining 30\% were confirmed to be objects showing no emission. The automatic method has improved these results (Alonso et al. 1995; Alonso 1996). In these studies we have compared the samples of ELGs obtained after visual and automatic searches in two fields. The new technique recovers nearly $80 \%$ of the objects detected by visual scanning. All of the objects not recovered by the automatic method are extended and very bright galaxies or galaxies with emission knots out of the nucleus. Moreover, with the automatic method we see an enhancement in the number of confirmed candidates selected. As shown in Alonso et al. (1995) and Alonso (1996), an increase of $24 \%$ over visual inspection has been reached in the total number of objects with confirmed $\mathrm{H} \alpha$ emission.

\section{PHYSICAL PARAMETERS}

We have given special attention to improving the extraction of information from our uncalibrated photographic plates. Several parameters have been obtained with intermediate accuracy for all the candidates. Table 2 presents full information for the objects of the third list of our survey. Column (1) lists the name of the galaxy according to the IAU rules. Equatorial coordinates for J2000.0 are given in columns (2) and (3). Column (4) presents an estimate of the $B$ magnitude obtained from our photographic plates (see $\S$ 4.4). In columns (5) and (6) we present the size at the $25 \mathrm{mag}$ arcsec $^{-2}$ isophote, in arcseconds, and the position angle (see $\S 4.2$ ), respectively. Estimated redshifts are listed in column (7) (see § 4.3). Column (8) gives a preliminary morphological classification derived by visual analysis of the Digitized Sky Survey $^{5}$ (DSS) images, and column (9) classifies each candidate into one of three categories according to the strength of the emission seen in the prism plates. Finally, column (10) lists previous designations of the candidates. (Finding charts from the DSS are included in Fig. 12.

The parameters listed in Table 2 are estimations, since spectroscopic and photometric studies have not been carried out. Because our uncalibrated plates do not permit us to directly obtain absolute parameters for the objects, we have performed several external calibrations by relating our photographic parameters with calibrated data for a small subsample of objects obtained from different catalogs. However, this approximation does not allow high accuracy,

\footnotetext{
${ }^{5}$ The Digitized Sky Surveys were produced at the Space Telescope Science Institute under U.S. Government grant NAG W-2166. The images of these surveys are based on photographic data obtained using the Oschin Schmidt Telescope on Palomar Mountain and the UK Schmidt Telescope. The plates were processed into the present compressed digital form with the permission of these institutions.
}

and some calibrations are not even possible. This is the case, for example, if we try to measure the equivalent width $\mathrm{EW}(\mathrm{H} \alpha)$ in our spectra, for which we will need a density-tointensity calibration, and, more importantly, a large sample of emission-line galaxies with known $\mathrm{EW}(\mathrm{H} \alpha)$ for each plate in order to obtain a well-sampled relation.

Next we briefly describe the procedure followed in obtaining each parameter.

\subsection{Astrometry}

We follow a standard procedure performed during the plate scan by MAMA in order to derive the constants for the plate astrometry. A third-order polynomial transformation is derived by using astrometric reference stars from the PPM catalog (Roeser \& Bastian 1991). The rms in the residuals of the astrometric reductions are about 0.3 , although large deviations can be achieved for very extended objects with several components. Columns (2) and (3) of Table 2 catalog the coordinates of the candidates with reference to the equinox $\mathbf{J} 2000.0$.

\subsection{Sizes and Position Angles}

During the object scanning process, we obtain the photographic major and minor axes and the position angle for each detected object by fitting an ellipse to pixels with a photographic density $3 \sigma$ over the local plate background. The size of a galaxy measured directly in the plate cannot be straightforwardly converted to size in arcseconds via the plate scale because of the photographic plate behavior. An ideal point of light is spread on the photographic emulsion over a wide area, and this effect grows with the source brightness. Hence, the size measured directly on the plate is a function of the real size of the source, the magnitude, and also the luminosity profile for an extended source. We have calibrated our photographic sizes with known galaxies, using the Catalogue of Principal Galaxies (PGC) in its electronic version, the Extragalactic Card Index System (ECIS) (Paturel et al. 1989). This catalog has the advantage of being homogeneous; sizes are all with reference to the isophote 25 mag $\operatorname{arcsec}^{-2}$ (Paturel et al. 1987). In Figure 2 we show the calibration derived for a single field; the other plates follow similar relations.

Figure 2 also shows that the instrumental MAMA sizes are systematically smaller than those obtained from the PGC. This is due to both the notably shallower depth of our photographic plates and the method used to obtain the sizes (thresholding the image $3 \sigma$ over the local background). Nevertheless, the calibrations derived allow us to estimate the size of our candidates to within an uncertainty of $20^{\prime \prime}$. Higher precision could be achieved with more homogeneous data. In Alonso (1996), an error of 2" was obtained calibrating with photometry for the UCM galaxies (Vitores et al. 1996b).

The position angle is adopted as the orientation of the fitted ellipse. There is no need for a calibration with external data, but a check was made using the same ECIS catalog cited above. This provided an estimation of the error, which was $8^{\circ}$ for $1 \sigma$. For very elongated galaxies the error should be notably smaller.

\subsection{Redshift}

The measurement of the redshift of the ELG candidates in our photographic plates requires a wavelength calibration of the spectra. This calibration involves obtaining 
TABLE 2

UCM SURVEY LIST 3

\begin{tabular}{|c|c|c|c|c|c|c|c|c|c|}
\hline $\mathrm{UCM}$ & R.A. (J2000.0) & Decl. (J2000.0) & $m_{B}{ }^{a}$ & $\begin{array}{c}\text { Size } \\
(\operatorname{arcsec})\end{array}$ & P.A. & Redshift $^{\mathrm{a}}$ & Morphology ${ }^{\mathbf{b}}$ & $\begin{array}{c}\text { OP } \\
\text { Emission }^{\mathrm{c}}\end{array}$ & Other Names \\
\hline $1345+2417 \ldots \ldots$ & 134806.3 & 240221 & 17.0 & $18 \times 14$ & 109 & 0.004 & $\mathrm{C}$ & $\mathrm{w}$ & \\
\hline $1345+2457 \ldots \ldots$ & 134736.5 & 244213 & 17.0 & $18 \times 17$ & 108 & 0.024 & $\mathrm{I}$ & $\mathrm{m}$ & \\
\hline $1346+2420 \ldots \ldots$ & 134918.2 & 240545 & 16.0 & $37 \times 18$ & 29 & 0.021 & I & $\mathrm{m}$ & \\
\hline $1347+2527 \ldots \ldots$ & 134937.6 & 251304 & 18.0 & $8 \times 7$ & 21 & 0.023 & $*$ & $\mathrm{~m}$ & \\
\hline $1348+2147 \ldots \ldots$ & 135117.8 & 213238 & $15.2^{*}$ & $35 \times 30$ & 112 & 0.025 & $S_{f}$ & $\mathrm{w}$ & KUG $1348+217$ \\
\hline $1348+2511 \ldots \ldots$ & 135020.2 & 245609 & 17.0 & $24 \times 12$ & 97 & 0.031 & $\mathrm{~S}$ & $\mathrm{~s}$ & IRAS F13480+2511 \\
\hline $1349+2151 \ldots \ldots$ & 135124.8 & 213619 & 17.0 & $14 \times 11$ & 82 & 0.038 & $*$ & $\mathrm{~s}$ & \\
\hline $1349+2152 \ldots \ldots$ & 135138.0 & 213739 & 16.5 & $36 \times 13$ & 69 & 0.031 & $\mathrm{~S}_{\mathrm{e}}$ & $\mathrm{m}$ & IRAS F13492+2152 \\
\hline $1350+2207 \ldots \ldots$ & 135320.3 & 215309 & 16.5 & $21 \times 13$ & 107 & 0.033 & I & $\mathrm{m}$ & \\
\hline $1350+2456 \ldots \ldots$ & 135226.5 & 244129 & 19.0 & $6 \times 4$ & 18 & 0.029 & $*$ & $\mathrm{~m}$ & \\
\hline $1350+2529 \ldots \ldots$ & 135224.9 & 251447 & 17.0 & $17 \times 12$ & 127 & 0.032 & $\mathrm{O}$ & $\mathrm{m}$ & \\
\hline $1351+2201 \ldots \ldots$ & 135325.7 & 214617 & 17.0 & $14 \times 11$ & 57 & 0.036 & $\mathrm{O}$ & $\mathrm{s}$ & \\
\hline $1351+2521 \ldots \ldots$ & 135338.7 & 250641 & 17.0 & $20 \times 11$ & 115 & 0.027 & $\mathrm{~S}_{\mathrm{e}}$ & $\mathrm{w}$ & \\
\hline $1352+2202 \ldots \ldots$ & 135458.8 & 214817 & 16.0 & $24 \times 19$ & 111 & 0.036 & $S_{f}$ & $\mathrm{~m}$ & IRAS F13526+2202 \\
\hline $1352+2256 \ldots \ldots$ & 135439.2 & 224140 & 17.0 & $22 \times 15$ & 0 & 0.023 & $S_{f}$ & $\mathrm{~m}$ & \\
\hline $1353+2507 \ldots \ldots$ & 135537.3 & 245237 & 17.0 & $16 \times 12$ & 58 & 0.030 & $\mathrm{O}$ & $\mathrm{w}$ & \\
\hline $1353+2517 \ldots \ldots$ & 135534.4 & 250259 & $16.09 *$ & $35 \times 21$ & 72 & $0.0295^{*}$ & $S_{f}$ & $\mathrm{w}$ & CGCG 132-048 \\
\hline $1353+2531 \ldots \ldots$ & 135536.1 & 251627 & 17.0 & $17 \times 9$ & 2 & 0.023 & $\mathrm{~S}_{\mathrm{e}}$ & $\mathrm{m}$ & \\
\hline $1353+2647 \ldots \ldots$ & 135538.0 & 263251 & $17.02 *$ & $16 \times 10$ & 99 & 0.009 & $S_{f}$ & $\mathrm{w}$ & NPM1G + 26.0340 \\
\hline $1355+2440 \ldots \ldots$ & 135737.8 & 242604 & 17.0 & $15 \times 10$ & 161 & 0.032 & $\mathrm{C}$ & $\mathrm{m}$ & \\
\hline $1356+2157 \ldots \ldots$ & 135836.2 & 214316 & 16.0 & $46 \times 16$ & 105 & 0.032 & $\mathrm{~S}_{\mathrm{e}}$ & $\mathrm{s}$ & \\
\hline $1356+2310 \ldots \ldots$ & 135824.7 & 225539 & 15.5 & $29 \times 21$ & 53 & $0.088^{*}$ & $\mathrm{~S}_{\mathrm{f}}$ & $\mathrm{m}$ & WAS 83 \\
\hline $1357+2614 \ldots \ldots$ & 140012.0 & 260021 & 18.5 & $12 \times 8$ & 172 & 0.020 & I & $\mathrm{m}$ & \\
\hline $1400+2304 \ldots \ldots$ & 140305.9 & 225026 & $17.41^{*}$ & $28 \times 13$ & 51 & 0.019 & $\mathrm{~S}$ & $\mathrm{w}$ & NPM1G + 23.0349 \\
\hline $1401+2602 \ldots \ldots$ & 140401.8 & 254744 & $14.9^{*}$ & $18 \times 15$ & 143 & $0.0330^{*}$ & $\mathrm{O}$ & $\mathrm{s}$ & WAS 89 \\
\hline $1402+2152 \ldots \ldots$ & 140453.0 & 213809 & $14.9^{*}$ & $38 \times 29$ & 118 & $0.0165^{*}$ & $\mathrm{~S}$ & $\mathrm{w}$ & MRK 0667 \\
\hline $1408+2543 \ldots \ldots$ & 141057.2 & 252948 & $14.43^{*}$ & $71 \times 64$ & 175 & $0.0316^{*}$ & $S_{f}$ & $\mathrm{w}$ & IC 4381 \\
\hline $1408+2547 \ldots \ldots$ & 141054.2 & 253314 & $15.9^{*}$ & $20 \times 16$ & 165 & $0.0312^{*}$ & $\mathrm{~S}_{\mathrm{f}}$ & $\mathrm{m}$ & WAS 90 \\
\hline $1408+2623 \ldots \ldots$ & 141028.5 & 260905 & 16.0 & $18 \times 17$ & 28 & $0.0297^{*}$ & $\mathrm{C}$ & $\mathrm{m}$ & IRAS F14081+2623 \\
\hline $1413+2317 \ldots \ldots$ & 141522.3 & 230350 & 17.0 & $22 \times 13$ & 95 & 0.023 & $\mathrm{~S}$ & $\mathrm{~m}$ & \\
\hline $1413+2446 \ldots \ldots$ & 141527.3 & 243226 & 20.0 & $7 \times 5$ & 48 & 0.024 & $*$ & $\mathrm{~m}$ & \\
\hline $1416+2202 \ldots \ldots$ & 141842.9 & 214909 & 15.0 & $78 \times 38$ & 91 & $0.084 *$ & $\mathrm{I}_{\mathrm{IP}}$ & $\mathrm{s}$ & UGC 09164 \\
\hline $1416+2300 \ldots \ldots$ & 141907.5 & 224619 & $17.15^{*}$ & $19 \times 18$ & 106 & 0.019 & $\mathrm{~S}$ & $\mathrm{~m}$ & $\mathrm{NPM} 1 \mathrm{G}+23.0356$ \\
\hline $1416+2543 \ldots \ldots$ & 141825.4 & 253004 & $15.7^{*}$ & $62 \times 18$ & 83 & $0.0150^{*}$ & $\mathrm{~S}_{\mathrm{e}}$ & $\mathrm{w}$ & KUG $1416+257$ \\
\hline $1418+2209 \ldots \ldots$ & 142046.6 & 215614 & $14.3^{*}$ & $16 \times 32$ & 120 & $0.0156^{*}$ & $\mathrm{~S}_{\mathrm{e}}$ & $\mathrm{m}$ & UGC 09182 \\
\hline $1419+2420 \ldots \ldots$ & 142152.9 & 240627 & $15.6^{*}$ & $30 \times 20$ & 118 & 0.020 & $\mathrm{~S}$ & $\mathrm{~m}$ & KUG $1419+243$ \\
\hline $1422+2321 \ldots \ldots$ & 142500.0 & 230732 & 16.0 & $23 \times 22$ & 17 & 0.017 & $S_{f}$ & $\mathrm{~s}$ & KUG $1422+233$ \\
\hline $1422+2450 \ldots \ldots$ & 142422.9 & 243652 & $14.11^{*}$ & $93 \times 45$ & 93 & $0.0171^{*}$ & $\mathrm{~S}_{\mathrm{B}}$ & $\mathrm{m}$ & NGC 5610 \\
\hline $1424+2515 \ldots \ldots$ & 142626.8 & 250147 & 17.0 & $14 \times 12$ & 134 & 0.022 & $\mathrm{O}$ & $\mathrm{w}$ & \\
\hline $1424+2537 \ldots \ldots$ & 142619.8 & 252403 & $15.5^{*}$ & $31 \times 23$ & 72 & 0.019 & $\mathrm{O}$ & $\mathrm{w}$ & KUG $1424+256$ \\
\hline $1424+2541 \ldots \ldots$ & 142615.5 & 252759 & 16.5 & $14 \times 13$ & 154 & 0.017 & $*$ & $\mathrm{w}$ & \\
\hline $1425+2146 \ldots \ldots$ & 142734.1 & 213325 & 17.0 & $21 \times 11$ & 106 & 0.030 & $\mathrm{~S}$ & $\mathrm{w}$ & \\
\hline $1426+2322 \ldots \ldots$ & 142910.9 & 230855 & 18.0 & $10 \times 10$ & 94 & 0.023 & $\mathrm{C}$ & $\mathrm{w}$ & \\
\hline $1427+2314 \ldots \ldots$ & 143011.0 & 230136 & $15.3^{*}$ & $39 \times 32$ & 81 & $0.0173^{*}$ & $\mathrm{~S}$ & $\mathrm{w}$ & MRK 0683 \\
\hline $1429+2145 \ldots \ldots$ & 143120.9 & 213210 & 16.5 & $25 \times 13$ & 94 & 0.018 & $\mathrm{~S}$ & $\mathrm{w}$ & \\
\hline $1431+2441 \ldots \ldots$ & 143320.3 & 242804 & 17.0 & $27 \times 12$ & 23 & 0.034 & S & $\mathrm{w}$ & \\
\hline $1432+2550 \ldots \ldots$ & 143506.5 & 253748 & 16.5 & $16 \times 12$ & 108 & 0.015 & S & $\mathrm{w}$ & \\
\hline $1435+2249 \ldots \ldots$ & 143810.4 & 223627 & 16.5 & $14 \times 14$ & 33 & 0.025 & S & $\mathrm{w}$ & \\
\hline $1436+2245 \ldots \ldots$ & 143821.1 & 223212 & 16.5 & $27 \times 18$ & 111 & 0.0405 & I & $\mathrm{w}$ & IRAS $14360+2245$ \\
\hline $1437+2148 \ldots \ldots$ & 143958.5 & 213559 & 16.5 & $36 \times 15$ & 99 & 0.031 & $\mathrm{~S}$ & $\mathrm{~m}$ & LSBC F580-06 \\
\hline $1438+2209 \ldots \ldots$ & 144115.8 & 215644 & 16.5 & $20 \times 19$ & 27 & 0.025 & $S_{f}$ & $\mathrm{~m}$ & $\mathrm{NPM} 1 \mathrm{G}+22.0467$ \\
\hline $1438+2239 \ldots \ldots$ & 144054.9 & 222708 & 17.0 & $22 \times 12$ & 167 & 0.014 & $\mathrm{~S}$ & $\mathrm{w}$ & IRAS F14386+2239 \\
\hline $1438+2307 \ldots \ldots$ & 144115.2 & 225432 & 17.5 & $13 \times 12$ & 4 & $0.0339 *$ & $\mathrm{~S}$ & $\mathrm{~m}$ & IRAS $14389+2307$ \\
\hline $1440+2521 \ldots \ldots$ & 144302.7 & 250908 & $16.16^{*}$ & $28 \times 14$ & 51 & $0.0319^{*}$ & $\mathrm{~S}$ & $\mathrm{~s}$ & UGC 09489 \\
\hline $1442+2248 \ldots \ldots$ & 144435.5 & 223538 & 17.5 & $14 \times 13$ & 11 & 0.025 & $\mathrm{~S}$ & $\mathrm{~m}$ & \\
\hline $1446+2312 \ldots \ldots$ & 144845.2 & 225934 & 15.5 & $45 \times 22$ & 35 & 0.008 & $\mathrm{~S}_{\mathrm{IP}}$ & $\mathrm{w}$ & IRAS F14465+2311 \\
\hline $1447+2535 \ldots \ldots$ & 144935.8 & 252252 & $14.34^{*}$ & $46 \times 45$ & 144 & $0.0339 *$ & $\mathrm{~S}_{\mathrm{f}}$ & $\mathrm{m}$ & UGC 09544 \\
\hline $1448+2248 \ldots \ldots$ & 145038.5 & 223631 & 17.5 & $12 \times 12$ & 164 & 0.034 & $\mathrm{C}$ & $\mathrm{w}$ & \\
\hline $1448+2256 \ldots \ldots$ & 145037.8 & 224406 & $15.7^{*}$ & $25 \times 22$ & 169 & $0.0215^{*}$ & $\mathrm{~S}$ & $\mathrm{~s}$ & MRK 1388 \\
\hline $1449+2559 \ldots \ldots$ & 145133.2 & 254658 & 19.0 & $7 \times 6$ & 47 & 0.021 & $\mathrm{C}$ & $\mathrm{m}$ & \\
\hline $1450+2342 \ldots \ldots$ & 145224.1 & 233041 & 17.5 & $14 \times 11$ & 120 & 0.034 & $\mathrm{~S}$ & $\mathrm{~m}$ & \\
\hline $1624+2359 \ldots \ldots$ & 162623.7 & 235241 & 17.0 & $20 \times 14$ & 98 & 0.040 & $\mathrm{~S}$ & $\mathrm{~m}$ & IRAS $16242+2359$ \\
\hline $1627+2433 \ldots \ldots$ & 162952.8 & 242639 & $15.5^{*}$ & $35 \times 34$ & 29 & $0.0375^{*}$ & I & $\mathrm{m}$ & VV 807 \\
\hline $1628+2453 \ldots \ldots$ & 163055.8 & 244649 & 17.0 & $20 \times 16$ & 140 & 0.026 & $\mathrm{~S}$ & $\mathrm{w}$ & \\
\hline
\end{tabular}


TABLE 2-Continued

\begin{tabular}{|c|c|c|c|c|c|c|c|c|c|}
\hline UCM & R.A. (J2000.0) & Decl. (J2000.0) & $m_{B}{ }^{a}$ & $\begin{array}{c}\text { Size } \\
(\operatorname{arcsec})\end{array}$ & P.A. & Redshift $^{\mathrm{a}}$ & Morphology ${ }^{\mathbf{b}}$ & $\begin{array}{c}\text { OP } \\
\text { Emission }^{\mathrm{c}}\end{array}$ & Other Names \\
\hline $1636+2632 \ldots \ldots$ & 163802.6 & 262704 & $15.93^{*}$ & $25 \times 22$ & 22 & 0.009 & $\mathrm{~S}$ & $\mathrm{~m}$ & $\mathrm{NPM} 1 \mathrm{G}+26.0432$ \\
\hline $1637+2417 \ldots \ldots$ & 163926.1 & 241159 & $17.48^{*}$ & $18 \times 15$ & 142 & 0.015 & $\mathrm{C}$ & $\mathrm{w}$ & $\mathrm{NPM} 1 \mathrm{G}+24.0414$ \\
\hline $1640+2238 \ldots \ldots$ & 164238.5 & 223310 & 17.5 & $32 \times 11$ & 37 & 0.011 & I & $\mathrm{m}$ & \\
\hline $1640+2510 \ldots \ldots$ & 164223.8 & 250507 & $14.8^{*}$ & $89 \times 28$ & 161 & $0.0227^{*}$ & $\mathrm{~S}$ & $\mathrm{~m}$ & UGC 10514 \\
\hline $1643+2213 \ldots \ldots$ & 164515.0 & 220822 & $15.7^{*}$ & $41 \times 23$ & 12 & $0.0316^{*}$ & $\mathrm{~S}$ & $\mathrm{w}$ & CGCG 138-069 \\
\hline $1647+2259 \ldots \ldots$ & 164923.9 & 225416 & 17.0 & $15 \times 11$ & 179 & 0.025 & * & $\mathrm{m}$ & \\
\hline $1650+2551 \ldots \ldots$ & 165231.8 & 254625 & $15.94 *$ & $28 \times 22$ & 139 & $0.0348^{*}$ & $\mathrm{~S}$ & $\mathrm{~s}$ & IRAS $16504+2551$ \\
\hline $1655+2532 \ldots \ldots$ & 165723.4 & 252757 & $16.61^{*}$ & $20 \times 16$ & 42 & 0.040 & $\mathrm{~S}$ & $\mathrm{~m}$ & $\mathrm{NPM} 1 \mathrm{G}+25.0438$ \\
\hline $1656+2413 \ldots \ldots$ & 165833.1 & 240851 & 18.0 & $17 \times 11$ & 163 & 0.019 & I & $\mathrm{m}$ & \\
\hline $1656+2450 \ldots \ldots$ & 165847.0 & 244624 & 17.5 & $38 \times 12$ & 152 & 0.025 & $\mathrm{~S}$ & $\mathrm{w}$ & \\
\hline $1701+2535 \ldots \ldots$ & 170305.1 & 253148 & 17.0 & $16 \times 13$ & 46 & 0.039 & $*$ & $\mathrm{~s}$ & \\
\hline $1701+2642 \ldots \ldots$ & 170348.2 & 263837 & 17.0 & $12 \times 11$ & 33 & 0.027 & * & $\mathrm{s}$ & \\
\hline $1702+2314 \ldots \ldots$ & 170459.9 & 231010 & 16.0 & $34 \times 25$ & 110 & $0.0304 *$ & $S_{f}$ & $\mathrm{w}$ & CGCG 139-033 \\
\hline $1706+2300 \ldots \ldots$ & 170852.3 & 225710 & 17.0 & $18 \times 10$ & 48 & 0.022 & $*$ & $\mathrm{~s}$ & \\
\hline $1710+2316 \ldots \ldots$ & 171245.9 & 231328 & $14.15^{*}$ & $53 \times 38$ & 166 & 0.034 & $\mathrm{~S}_{\mathrm{B}}$ & $\mathrm{s}$ & NGC 6315 \\
\hline $1711+2427 \ldots \ldots$ & 171312.3 & 242342 & 19.0 & $14 \times 6$ & 71 & 0.035 & I & $\mathrm{w}$ & \\
\hline $1712+2305 \ldots \ldots$ & 171425.5 & 230139 & 17.0 & $20 \times 12$ & 153 & 0.030 & $\mathrm{~S}$ & $\mathrm{w}$ & \\
\hline $1712+2306 \ldots \ldots$ & 171430.0 & 230338 & $15.1^{*}$ & $34 \times 27$ & 109 & $0.0295^{*}$ & $\mathrm{~S}$ & $\mathrm{w}$ & ARK 520 \\
\hline $1714+2442 \ldots \ldots$ & 171654.3 & 243854 & 18.0 & $14 \times 13$ & 32 & 0.021 & I & $\mathrm{m}$ & \\
\hline $1714+2541 \ldots \ldots$ & 171642.6 & 253803 & $15.7^{*}$ & $39 \times 22$ & 115 & 0.023 & $\mathrm{~S}$ & $\mathrm{w}$ & CGCG 140-004 \\
\hline $1717+2428 \ldots \ldots$ & 171934.6 & 242531 & 18.0 & $12 \times 12$ & 15 & 0.028 & $\mathrm{O}$ & $\mathrm{s}$ & \\
\hline $1717+2458 \ldots \ldots$ & 171956.6 & 245557 & 17.0 & $29 \times 18$ & 126 & 0.019 & $\mathrm{~S}_{\mathrm{B}}$ & $\mathrm{s}$ & \\
\hline $1721+2326 \ldots \ldots$ & 172329.0 & 232336 & 17.5 & $25 \times 22$ & 23 & 0.005 & $\mathrm{I}_{\mathrm{IP}}$ & $\mathrm{s}$ & \\
\hline $1722+2500 \ldots \ldots$ & 172445.4 & 245817 & $14.2^{*}$ & $68 \times 37$ & 144 & $0.0276^{*}$ & $\mathrm{~S}$ & $\mathrm{w}$ & UGC 10837 \\
\hline $1722+2656 \ldots \ldots$ & 172450.7 & 265332 & 17.0 & $29 \times 26$ & 135 & 0.031 & $\mathrm{~S}_{\mathrm{f}}$ & $\mathrm{w}$ & \\
\hline $1723+2556 \ldots \ldots$ & 172548.6 & 255333 & 18.0 & $17 \times 11$ & 161 & 0.014 & $\mathrm{C}_{\mathrm{IP}}$ & $\mathrm{w}$ & \\
\hline $1725+2653 \ldots \ldots$ & 172747.0 & 265116 & $15.74^{*}$ & $31 \times 26$ & 62 & $0.0296^{*}$ & $\mathrm{~S}$ & $\mathrm{w}$ & VV 389 \\
\hline $1726+2339 \ldots \ldots$ & 172818.8 & 233727 & 15.5 & $30 \times 23$ & 121 & 0.030 & $\mathrm{~S}$ & $\mathrm{~s}$ & CGCG 140-031 \\
\hline $1727+2549 \ldots \ldots$ & 172933.6 & 254648 & 18.0 & $12 \times 12$ & 25 & 0.021 & I & $\mathrm{m}$ & \\
\hline $1729+2548 \ldots \ldots$ & 173114.8 & 254620 & 18.5 & $13 \times 12$ & 43 & 0.020 & I & $\mathrm{w}$ & \\
\hline $1732+2414 \ldots \ldots$ & 173449.5 & 241229 & 18.5 & $10 \times 8$ & 136 & 0.019 & $\mathrm{C}$ & $\mathrm{w}$ & \\
\hline $1732+2509 \ldots \ldots$ & 173449.7 & 250744 & 17.5 & $21 \times 12$ & 121 & 0.022 & IP & $\mathrm{m}$ & \\
\hline $1733+2441 \ldots \ldots$ & 173538.6 & 243939 & 18.0 & $16 \times 8$ & 31 & 0.018 & $\mathrm{C}$ & $\mathrm{w}$ & \\
\hline $1733+2554 \ldots \ldots$ & 173514.3 & 255231 & 17.5 & $11 \times 9$ & 11 & 0.028 & $\mathrm{C}$ & $\mathrm{s}$ & \\
\hline $1734+2219 \ldots \ldots$ & 173629.6 & 221715 & $16.89^{*}$ & $21 \times 16$ & 94 & 0.015 & $\mathrm{~S}$ & $\mathrm{~m}$ & NPM1G + 22.0588 \\
\hline $1734+2322 \ldots \ldots$ & 173636.3 & 232108 & $17.61^{*}$ & $20 \times 14$ & 73 & 0.025 & $\mathrm{~S}$ & $\mathrm{w}$ & $\mathrm{NPM} 1 \mathrm{G}+23.0458$ \\
\hline $1735+2617 \ldots \ldots$ & 173708.0 & 261601 & 19.0 & $8 \times 6$ & 46 & 0.014 & $\mathrm{C}$ & $\mathrm{m}$ & \\
\hline $1735+2622 \ldots \ldots$ & 173748.0 & 262118 & $16.51^{*}$ & $21 \times 17$ & 155 & 0.022 & $\mathrm{~S}$ & $\mathrm{w}$ & $\mathrm{NPM} 1 \mathrm{G}+26.0460$ \\
\hline $1736+2458 \ldots \ldots$ & 173827.2 & 245713 & $15.1^{*}$ & $54 \times 22$ & 134 & $0.0209 *$ & $\mathrm{~S}$ & $\mathrm{w}$ & UGC 10926 \\
\hline $1738+2544 \ldots \ldots$ & 174014.4 & 254305 & 18.0 & $11 \times 10$ & 35 & 0.018 & I & $\mathrm{m}$ & \\
\hline $1739+2637 \ldots \ldots$ & 174141.2 & 263618 & 17.5 & $14 \times 12$ & 149 & 0.030 & $\mathrm{~S}$ & $\mathrm{~m}$ & \\
\hline $1739+2639 \ldots \ldots$ & 174146.8 & 263800 & 17.5 & $26 \times 10$ & 124 & 0.023 & $\mathrm{~S}$ & $\mathrm{~m}$ & \\
\hline $1740+2210 \ldots \ldots$ & 174240.7 & 220913 & 16.5 & $18 \times 13$ & 138 & 0.043 & $\mathrm{~S}$ & $\mathrm{w}$ & \\
\hline $1740+2351 \ldots \ldots$ & 174245.1 & 235030 & 17.5 & $22 \times 11$ & 94 & 0.028 & $\mathrm{~S}$ & $\mathrm{w}$ & \\
\hline $1742+2343 \ldots \ldots$ & 174500.2 & 234222 & 19.0 & $10 \times 9$ & 169 & 0.014 & $\mathrm{C}$ & $\mathrm{s}$ & \\
\hline $1742+2634 \ldots \ldots$ & 174440.1 & 263325 & 19.5 & $10 \times 8$ & 133 & 0.025 & $\mathrm{C}$ & $\mathrm{w}$ & \\
\hline $1744+2629 \ldots \ldots$ & 174626.4 & 262853 & 19.0 & $9 \times 8$ & 48 & 0.024 & $\mathrm{C}$ & $\mathrm{m}$ & \\
\hline $1745+2235 \ldots \ldots$ & 174718.5 & 223441 & 17.0 & $17 \times 12$ & 40 & 0.025 & $\mathrm{~S}$ & $\mathrm{w}$ & \\
\hline $1746+2412 \ldots \ldots$ & 174847.1 & 241118 & 17.0 & $28 \times 15$ & 87 & 0.028 & I & $\mathrm{s}$ & \\
\hline
\end{tabular}

NoTE.- Units of right ascension are hours, minutes, and seconds, and units of declination are degrees, arcminutes, and arcseconds.

${ }^{a}$ Data obtained from the literature are marked with an asterisk.

${ }^{b}$ Morphological types: C, compact; I, irregular; $\mathrm{O}$, oval; IP, interacting pair; asterisk (*), stellar-like; and $\mathrm{S}$, spiral $\left(\mathrm{S}_{\mathrm{f}}\right.$, near face-on; $\mathrm{S}_{\mathrm{e}}$, near edge-on; $\mathrm{S}_{\mathrm{B}}$, barred spiral).

${ }^{c}$ Visual estimation of the $\mathrm{H} \alpha$ emission (w, weak; m, medium; s, strong).

the prism dispersion and further requires knowledge of a reference point at a specific wavelength for each spectrum.

The prism disperses the light by means of the changing refractive index with the following dependence on wavelength, in first order:

$$
n(\lambda)=a+\frac{b}{\lambda^{2}},
$$

where $a$ and $b$ are constants. Thus, the position of a certain spectral feature in the objective-prism image will follow the same dependency,

$$
x(\lambda)=A+\frac{B}{\lambda^{2}} .
$$

Here, the $A$ constant depends on the reference point we choose, while the $B$ constant is only related to the characteristics of the prism.

A test plate was taken in order to obtain the dispersion 


\section{Size calibration}

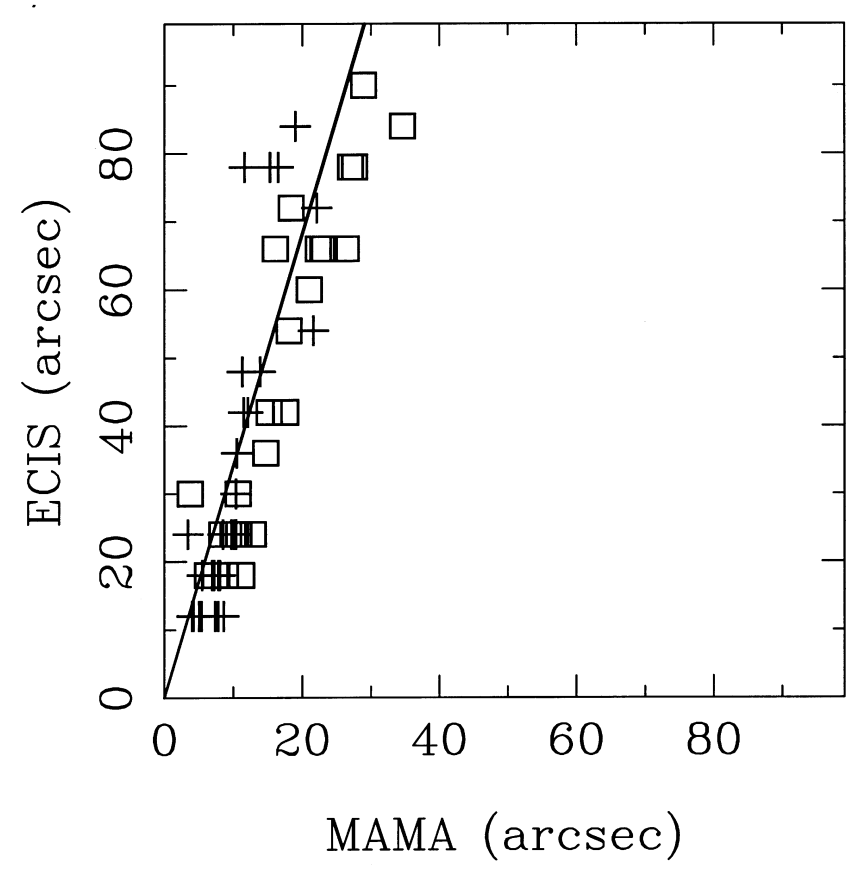

FIG. 2.-Relationship between the sizes for a small sample of galaxies taken from the ECIS catalog and those measured in our direct plates. Major and minor axis values are represented by squares and crosses, respectively. The straight line shows the best fit. See $\S 4.2$ for details.

curve for the $4^{\circ}$ objective prism of the Calar-Alto Schmidt Telescope. The configuration used was a IIIaF emulsion without a filter. The prism spectra of several bright stars were registered with a short exposure, covering a spectral range from the blue atmospheric cutoff until $6850 \AA$, the limit set by the sharp red cutoff of the IIIaF emulsion. The dispersion curve was obtained by measuring the position of the strong absorption Balmer lines of A stars. Fitting the data to the expression (Alonso 1996; see also Fig. 3)

$$
\frac{d \lambda}{d x}=-\frac{\lambda^{3}}{2 B}
$$

yields a value of $B=(-713 \pm 2) \times 10^{8} \AA^{2} \mu \mathrm{m}$. To measure the wavelength of the $\mathrm{H} \alpha$ feature in the prism spectra we also need a reference point. The emulsion red cutoff at $\sim 6850 \AA$ cannot be used because of its high dependency with brightness, color, and size. We have instead used the astrometric reduction to obtain a constant reference point for all the spectra. This point is obtained by converting the position of the object from the direct to the prism plate. We do not know the wavelength of this reference point, but it will be the same for all the spectra, even for different plates, where we have applied the same astrometric reduction, and it will not be affected by the effects previously noted (size, brightness, and color). Only when the emission comes from the center of the galaxy does the method clearly fail. Two identical galaxies showing $\mathrm{H} \alpha$ emission, the first in the nucleus and the second in an external knot, would show the emission line in different locations of their prism spectra with respect to a same reference point obtained from the plate-to-plate transformation. This fact would therefore yield an erroneous value of the redshift for the second galaxy.
CAHA Schmidt Telescope. $4^{\circ}$ Prism

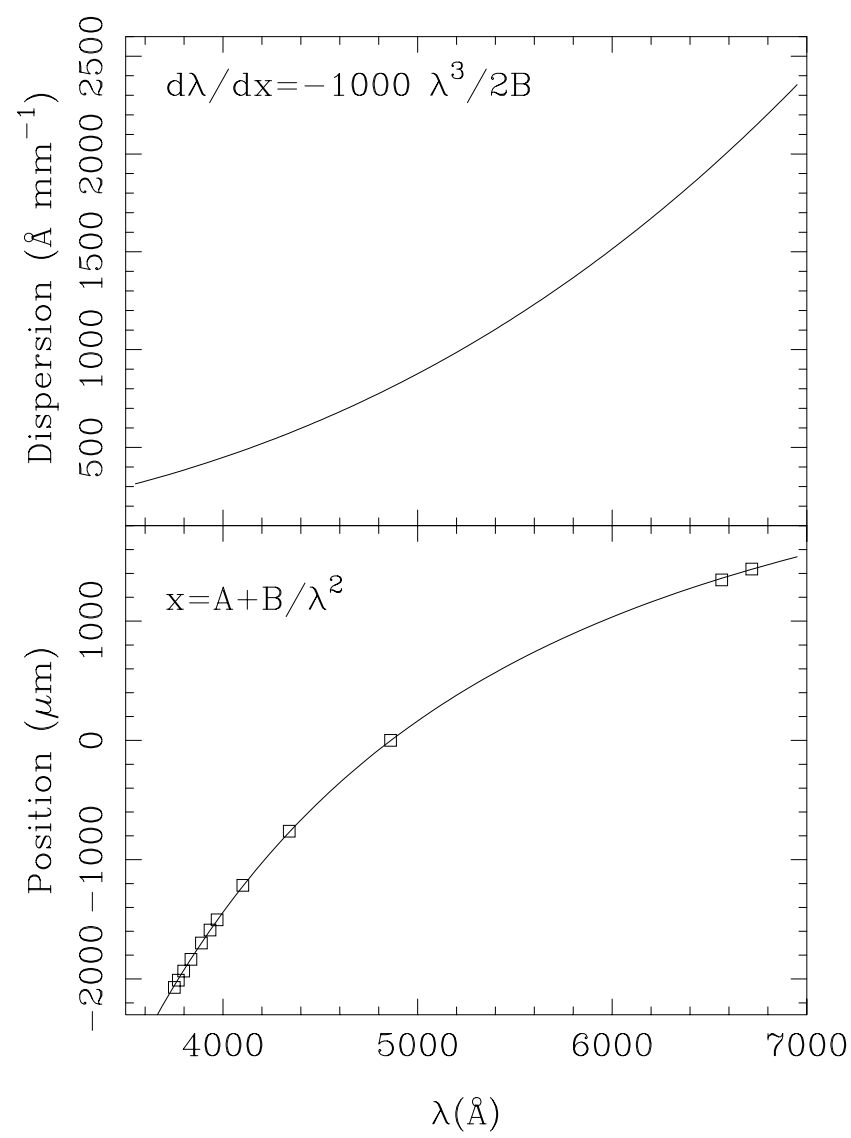

FIG. 3.-Dispersion curve for the $4^{\circ}$ objective prism of the Schmidt Telescope at the Calar Alto Observatory. The bottom plot shows the position of some spectral features measured in A-type stars relative to the $\mathrm{H} \beta$ line vs. wavelength. The curve displays the best fit, assuming a refraction index law $n(\lambda)=a+b / \lambda^{2}$. The top diagram shows the dispersion dependence with wavelength.

In order to obtain the wavelength of this reference point (that is, the value of the constant $A$ ), we represent the redshift $(z)$ of known galaxies with respect to the $\mathrm{H} \alpha$ position in the prism spectrum, relative to the reference point $(\Delta x)$. This relation must follow the expression

$$
z=\frac{\sqrt{B /[\Delta x(\mu m)-A]}-\lambda_{0}}{\lambda_{0}},
$$

where $\lambda_{0}$ is $6562.8 \AA$ if we are working with the $\mathrm{H} \alpha$ line. Therefore, we can calculate the value of $A$ by applying an algorithm of minimum squares to the data. The value of this constant, obtained separately for the nine fields, yields the same results inside the $1 \sigma$ error bar, confirming the procedure.

Assuming an uncertainty of 1 pixel in the determination of the center of the line, and taking into account the rms in the calibration fit, the typical error in the computation of the redshift results is 0.004 for $1 \sigma$ (i.e., $1200 \mathrm{~km} \mathrm{~s}^{-1}$ ). In Figure 4 we present the data obtained by applying the method just described to a small subsample of galaxies with known redshifts in order to test our procedure. The galaxies showing large deviations are explained by noting that the method may fail when the galaxy is very extended and the emission feature comes from an external knot. This is the case for UCM $1436+2245$ (IRAS $14360+2245$ ), with a red- 


\section{Redshift calibration}

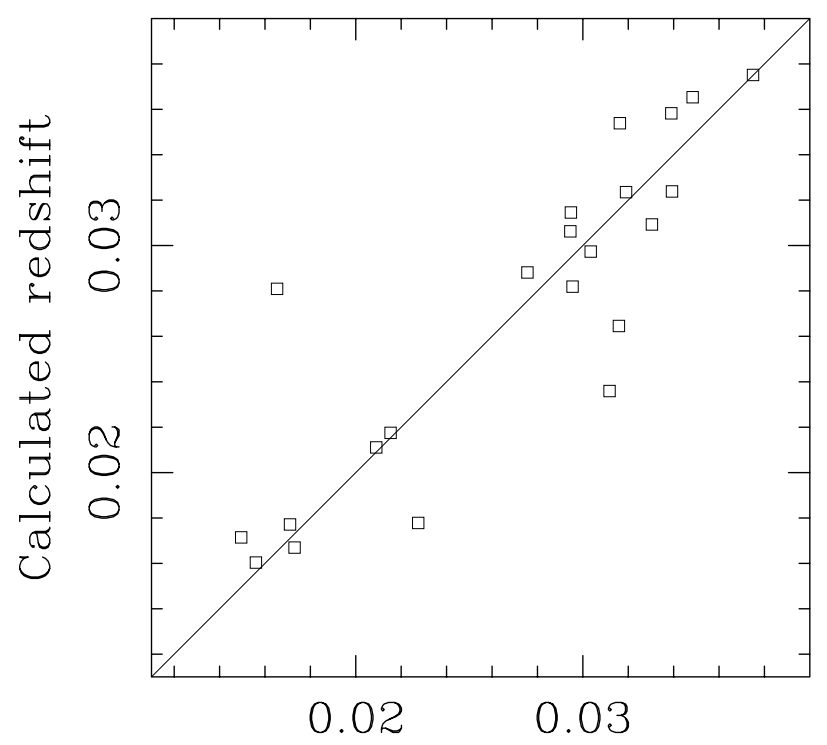

Known redshift

FIG. 4.- Relationship between the redshift obtained from the prism spectra, applying the method described in $\S 4.3$, and those recovered from the literature for a small subsample of ELGs.

shift of 0.04 (Fisher et al. 1995). Our method gives an estimate of $z=0.017$, owing to the presence of a double-component emission, probably originating in different knots. Column (7) of Table 2 presents the redshift obtained for noncataloged candidates. Data marked with an asterisk are obtained from literature.

Obviously, these redshifts are based on the assumption that the emission feature registered in the prism spectra is the $\mathrm{H} \alpha$ line. This conjecture is based on the fact that none of the confirmed emission-line galaxies from the previous UCM Lists 1 and 2 were revealed to be distant galaxies with their emission lines redshifted to our spectral coverage. In addition, while a galaxy with a magnitude of $M^{*}=-21.4$ located at $z=0.3$ will show the [O III] 5007 line in the $\mathrm{H} \alpha$ region, the apparent magnitude would be notably fainter than the limit of our prism plates $\left(m_{r} \approx 18\right)$.

\subsection{Magnitudes}

The direct plates were obtained with an instrumental response near the $B$ band of the Johnson system, and therefore we are interested in measuring the magnitudes of our ELG sample. Our plates are not calibrated, but we can relate the photographic flux, the sum of the density for all the pixels of the image, to the $B$ magnitude for objects recovered from databases. This relation works fine for stellar objects ( $\sigma \approx 0.1 \mathrm{mag}$ ), but it is not straightforward to apply it to extended objects because of the dependence with the size of the source. Using both photographic density and size it is possible to estimate the magnitudes, but with larger errors, up to $0.5 \mathrm{mag}$ for very extended galaxies.

Several studies have been published that have achieved better accuracy using photographic plates, but in general all of them require calibration spots (Surace \& Comte 1994); otherwise the calibration gives good results only in photometry for stellar-like objects (Kroll \& Neugebauer 1993; Berger et al. 1991; Mohan \& Crézé 1987). In general, the photometry of extended sources has several sources of error, which are sometimes hard to correct for (see de Vaucouleurs 1984).

The $B$ magnitudes listed in column (4) of Table 2 are derived using the method described above, and should be used only as estimates for statistical or observational purposes. Only values for compact galaxies can be taken with greater confidence.

\section{SELECTION EFFECTS}

One of the major problems encountered when working with photographic plates is that the characteristics of each plate can vary notably with respect to the others, even when special care is taken to repeat the entire observational setup, making it almost impossible to obtain a homogeneous sample of galaxies. In order to understand the biases of our observational procedure, we have compared the samples of galaxies obtained in our survey with those recovered using different observational configurations. This task is described in detail in Zamorano et al. $(1994,1996)$ for UCM Lists 1 and 2, and in $\S 7$ of the present paper for the new List 3.

Nevertheless, in this section we use the spectroscopic data derived from the two previous lists of the UCM survey (Gallego et al. 1995) with the aim of investigating the observational biases of our instrumental configuration. This analysis (Alonso 1996) shows that neither the equivalent width (EW) of the emission nor the flux of the $\mathrm{H} \alpha$ line alone are the parameters that control the detection limit in prism surveys. Moss, Whittle, \& Irwin (1988) introduced an auxiliary parameter, defined as EW $\times$ Flux, and showed that it accurately defined the observational limit of their work, an objective-prism survey in the red region of the spectrum, at a reciprocal dispersion of $400 \AA \mathrm{mm}^{-1}$. We have also used this parameter in our survey. In Figure 5 we show the histogram of values EW $(\AA) \times$ Flux $\left(\mathrm{ergs} \mathrm{s}^{-1} \mathrm{~cm}^{-2}\right)$ for the sample of UCM galaxies from Lists 1 and 2. We can conclude that the UCM survey is able to identify emission-line galaxies with a value of $\log (\mathrm{EW} \times$ Flux $)>-13$. Only four objects have lower values of this parameter. Nevertheless,

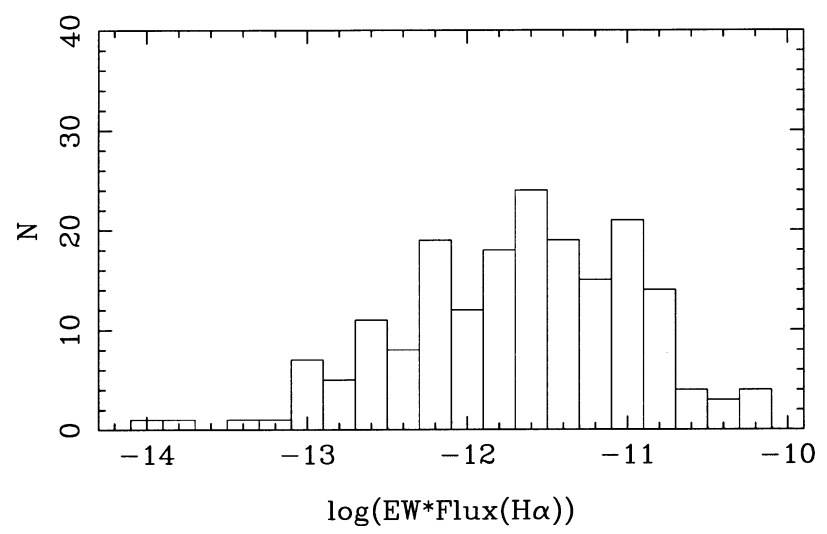

FIG. 5.- Histogram of values EW $\times$ Flux (of the $\mathrm{H} \alpha$ emission line) for the sample of galaxies from UCM Lists 1 and 2. 


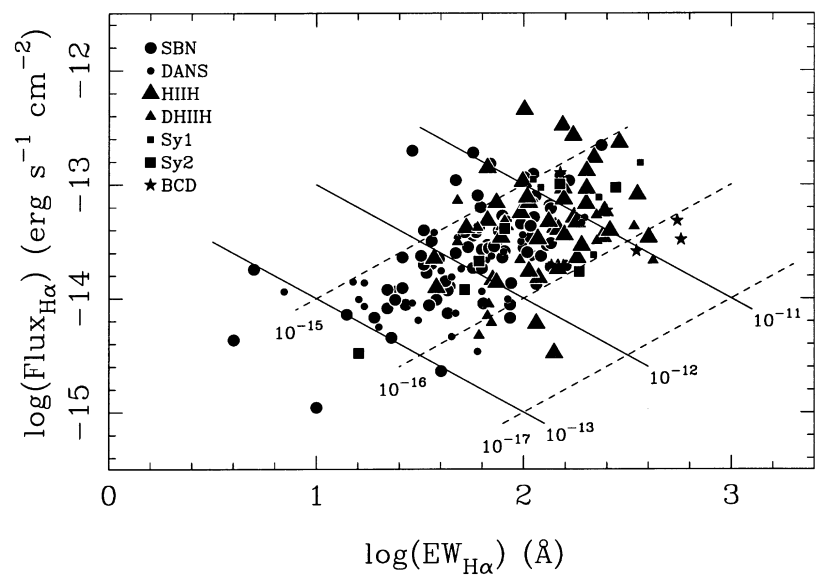

FIG. 6.- $\mathrm{H} \alpha$ Flux vs. EW for the sample of galaxies from UCM Lists 1 and 2 . Solid lines connect the points with the parameter EW $\times$ Flux constant. Dashed lines show the location of galaxies with continuum flux (in ergs $\mathrm{s}^{-1} \mathrm{~cm}^{-2} \AA^{-1}$ ) constant.

three of them have redshifts larger than the limit imposed by the emulsion sensitivity, making it impossible to register the $\mathrm{H} \alpha$ emission. These objects must be considered as serendipitous identifications.

In Figure 6 we show the distribution of the UCM objects from Lists 1 and 2 in a $\log$ (Flux) versus $\log (\mathrm{EW})$ plot. The data have been obtained from the work of Gallego et al. (1996). The plot shows the behavior of a sample obtained by prism plates. It also shows a lack of galaxies in the upper left and lower right corners. These two regions belong to very bright and faint galaxies, respectively. Therefore, the diagram shows that, in addition to a limiting magnitude, there exists a deficiency of bright galaxies due to saturation of the photographic plates. We cannot represent the location of the galaxies obtained in this List 3 until spectroscopic observations are performed. Nevertheless, these effects are due not to the visual or automatic procedure for selecting the candidates, but to the intrinsic characteristics of the photographic emulsion. This is why we expect a similar response for the galaxies identified in the present list.

\section{STATISTICAL PROPERTIES ${ }^{6}$}

\subsection{Apparent Magnitudes}

The histogram of apparent magnitudes presented in Figure 7 informs us about the depth of the survey. Nearly all the galaxies lie between 16 and 18 in $B$ magnitude, with a few reaching magnitude 20 . The mean apparent $B$ magnitude for the sample is 16.8. This histogram also shows a known bias of this technique: it is unable to detect bright galaxies, because they appear saturated in the photographic plates. This occurs, for example, for several known galaxies, such as NGC $5637\left(m_{B}=14.7\right)$ and WAS $82\left(m_{B}=15.5\right)$. We also present the histogram of Gunn-Thuan $r$ magnitudes obtained from the UCM Lists 1 and 2. Assuming a mean color $B-r=0.65$ for the UCM sample of galaxies, a typical value for Scd galaxies (Fukugita, Shimasaku, \& Ich-

\footnotetext{
${ }^{6}$ From here on, we adopt values of $H_{0}=50 \mathrm{~km} \mathrm{~s}^{-1} \mathrm{Mpc}^{-1}$ and $q_{0}=0.5$.
}

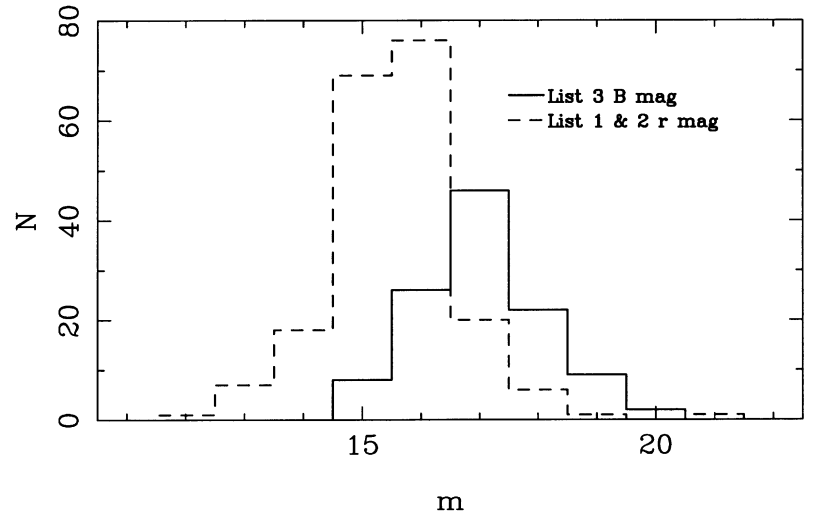

FIG. 7.-Histograms of apparent magnitudes from UCM Lists 1 and 2 (dashed line; Gunn-Thuan $r$ magnitudes) and UCM List 3 (solid line; $B$ magnitudes).

ikawa 1995; Vitores et al. 1996b), the comparison points out that UCM List 3 seems to be slightly deeper that the previous ones, although the lack of precision in our $B$ magnitudes prevents us from extending these conclusions. We note that the large number of galaxies in the histogram for UCM Lists 1 and 2 is due to the greater area explored compared to List 3.

\subsection{Absolute Magnitudes}

Figure 8 shows the histogram in absolute magnitude. The mean absolute magnitude is $M_{B}=-18.9$. This histogram is less symmetrical than the distribution of $M_{r}$ derived from Lists 1 and 2 (Vitores et al. 1996b). Our coverage of the low-luminosity end of the luminosity function is far better than in the previous UCM lists. It is worth noting than galaxies with $M_{B}$ as faint as -15 have been detected. Such a high percentage of low-luminosity galaxies has been also reached by the University of Michigan survey for emissionline galaxies (MacAlpine, Smith, \& Lewis 1977).

\subsection{Surface Brightness}

We have computed the mean surface brightness using the apparent blue magnitude and the size referred to the iso-

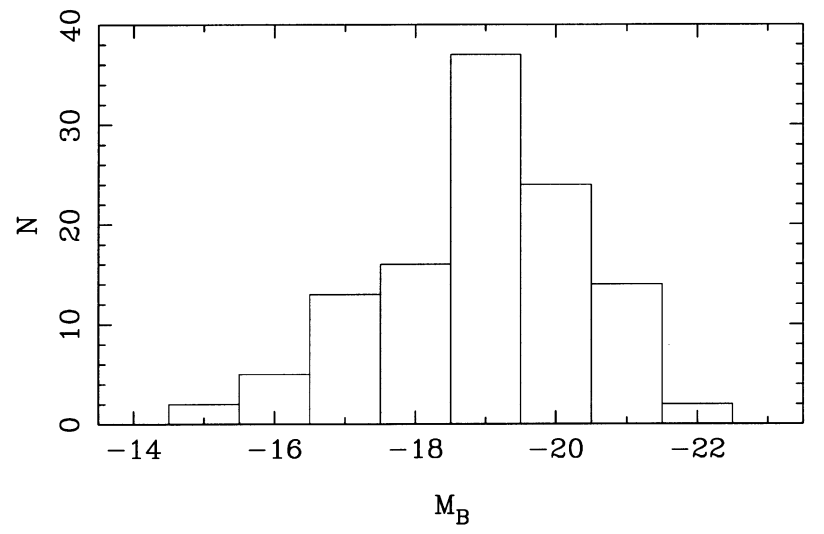

FIG. 8.- Histogram of absolute $B$ magnitudes obtained for UCM List 3. 


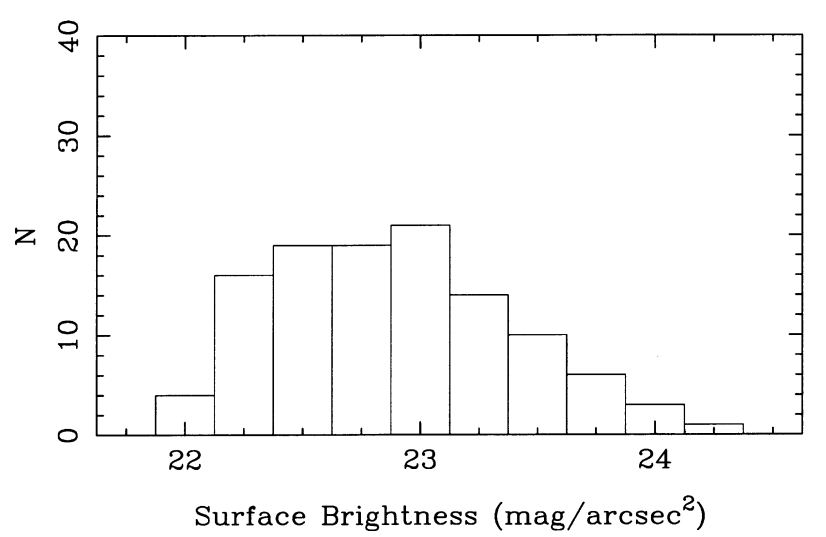

FIG. 9.- Histogram of mean $B$ surface brightness inside the 25 mag $\operatorname{arcsec}^{-2}$ isophote.

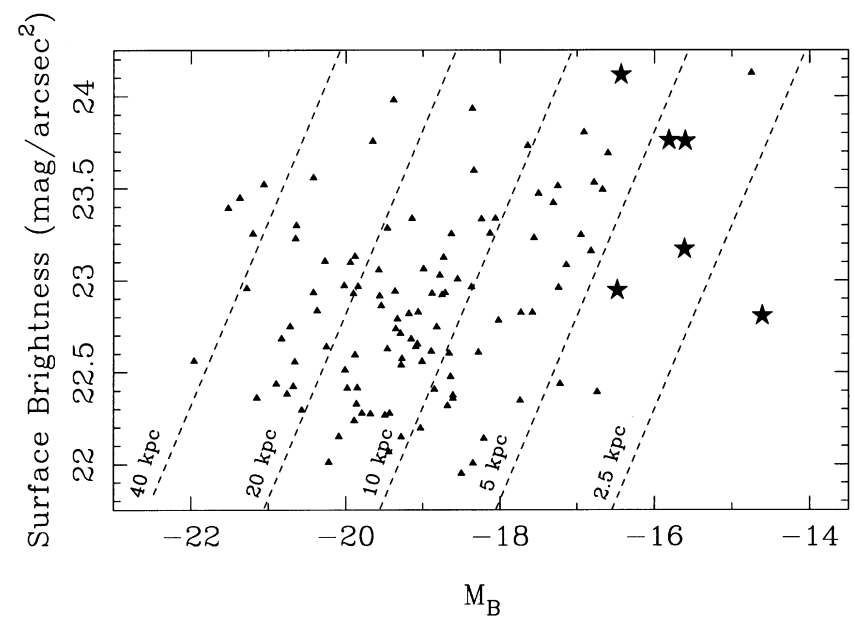

FIG. 10.- Surface brightness vs. absolute magnitude in the $B$ band. Dashed lines represent galaxies with constant diameter size. Blue compact galaxy candidates are marked with stars.
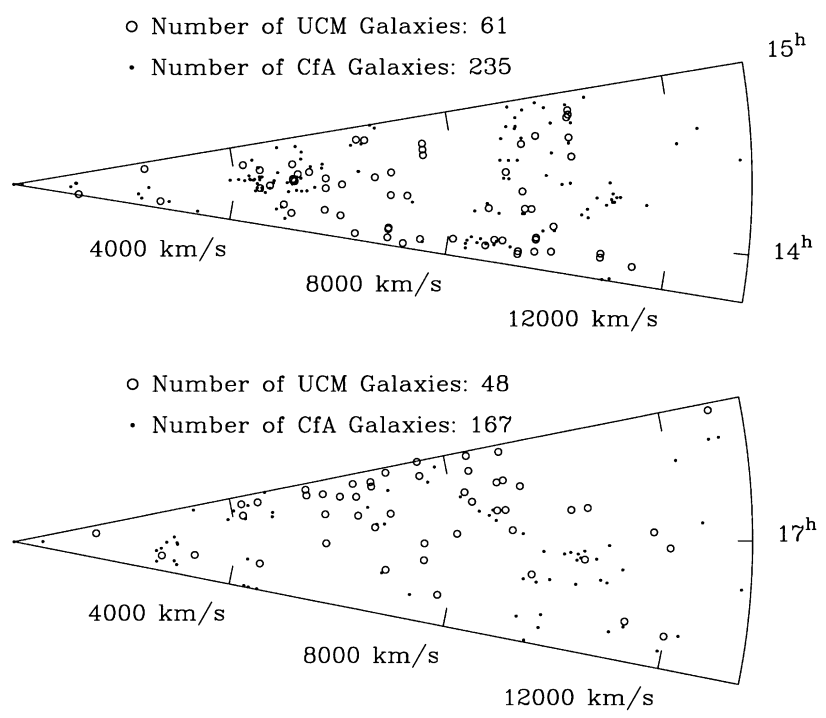

FIG. 11.- Redshift-position diagrams for candidates of UCM List 3. The slices cover $6^{\circ}$ centered at 24.5 in declination. Solid dots represent galaxies from the CfA survey, while open circles show the UCM galaxies. phote $25 \mathrm{mag} \operatorname{arcsec}^{-2}$. The mean surface brightness of our sample is distributed almost uniformly from 21.5 to 24.5 mag $\operatorname{arcsec}^{-2}$, with a mean value of 22.8 (see Fig. 9). Lists 1 and 2 yielded a mean surface brightness of $22 \mathrm{mag} \operatorname{arcsec}^{-2}$ in the Gunn-Thuan $r$ band (Vitores et al. 1996b). The precision of our data prevents us from extending this study to filter or color differences.

In Figure 10 we plot $B$ magnitude versus surface brightness for the sample. The BCD type galaxies are mainly distinguished by their low luminosity and high surface brightness. The right side of the plot is populated by such galaxies. Considering an upper limit of $M_{B}>-18$ (Thuan $\&$ Martin 1981) for a BCD galaxy, we regard those galaxies with a compact aspect and a more conservative $M_{B}>$ -16.5 as good candidates to be BCD galaxies. Following these criteria, the most probable candidates for BCD galaxies are: UCM 1345+2417, UCM 1413+2446, UCM $1449+2559$, UCM $1735+2617$, UCM $1742+2343$, and UCM $1742+2634$. Other type of galaxies found in this region are irregular galaxies with strange morphologies, such as comet-like shapes, double components, etc. UCM $1640+2238$, UCM $1721+2326$, and UCM $1723+2556$ are such galaxies, and so were not included in the BCD sample.

\subsection{Spatial Distribution}

In Figure 11 we show the spatial distribution of our sample for both the $14^{\mathrm{h}}$ and the $16^{\mathrm{h}}$ regions. The solid dots represent galaxies from the CfA survey (Huchra, Geller, \& Corwin 1995), which have been plotted to show the normal galaxy distribution. It can be seen that several clustering structures appear in the CfA data. A well-defined cluster is located at $5000 \mathrm{~km} \mathrm{~s}^{-1}$, and there are three more at 9000 , 9500 , and $11,000 \mathrm{~km} \mathrm{~s}^{-1}$ (beyond the UCM instrumental limit in redshift) in the $14^{\mathrm{h}}$ field. In the $17^{\mathrm{h}}$ sector, only one cluster is observed at $10,000 \mathrm{~km} \mathrm{~s}^{-1}$. At this redshift we detect no galaxies.

Figure 11 shows the locations of the UCM galaxies with open circles. They nearly follow the distribution of the CfA survey, although less clustering seems to appear. Preliminary analysis points to the idea that preferred galaxy location could be related to physical size.

\section{COMPARISON WITH OTHER SURVEYS}

The UCM Survey has found 113 candidates in the 9 fields $\left(189 \mathrm{deg}^{2}\right)$ of List 3. (Finding charts from the DSS are shown in Fig. 12.) The overall density is around 0.59 candidates per $\mathrm{deg}^{2}$, slightly greater that the value derived from UCM Lists 1 and 2 . It is worth noting that the actual density of galaxies with $\mathrm{H} \alpha$ emission (excluding candidates with no emission) is $\sim 0.4$ for the first two lists (Gallego et al. 1996). One of the properties of the automatic method is a better success rate of detection (Alonso 1996), which will be determined for this survey with follow-up spectroscopy. Therefore, this value is consistent with an improvement in the number of galaxies detected. Although we have lost some bright and extended galaxies, at fainter magnitudes the automatic procedure is more sensitive, providing an increase in the number of galaxies per $\mathrm{deg}^{2}$.

In Lists 1 and 2, some comparisons were made between the samples detected with different surveys and observational techniques, and that obtained by us. The conclusions we derived do not change when the new UCM fields surveyed are added to the comparison. Following Kinman 


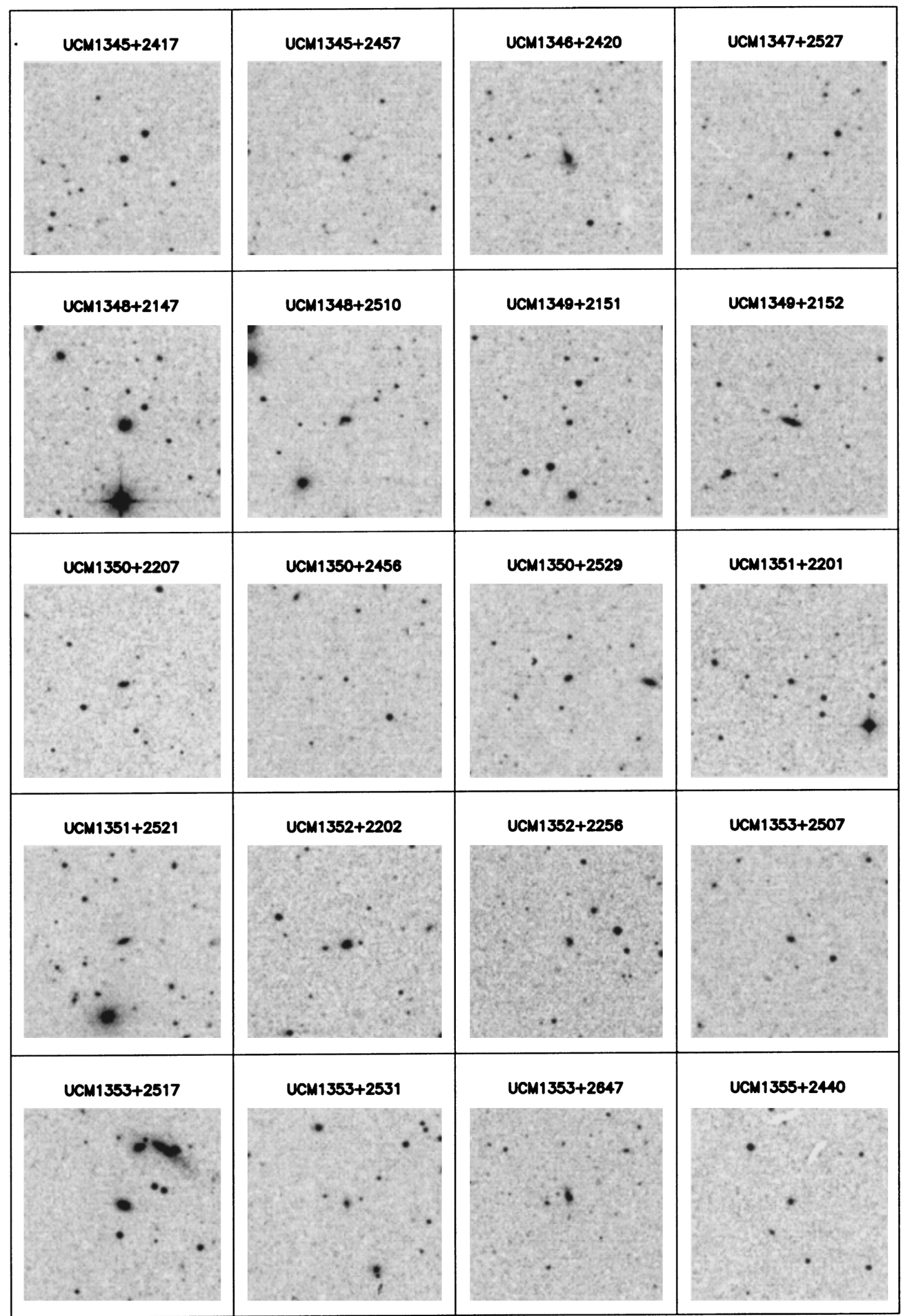

FIG. 12.-Finding charts for UCM galaxies of List 3. Each field covers a square $5^{\prime}$ wide. Object is centered in the frame.

(1984), the Catalogue of Galaxies and Clusters of Galaxies (CGCG) galaxies have been used as the reference sample of galaxies in the field. Only 26 galaxies out of 242 galaxies with known redshift $z \leq 0.04$ have been found with emis- sion. The ratio is $\sim 11 \%$, similar to the $13 \%$ found in the previous lists.

The UCM List 3 fields do not overlap completely with the KUG survey (Kiso Ultraviolet-excess Galaxies; Takase 


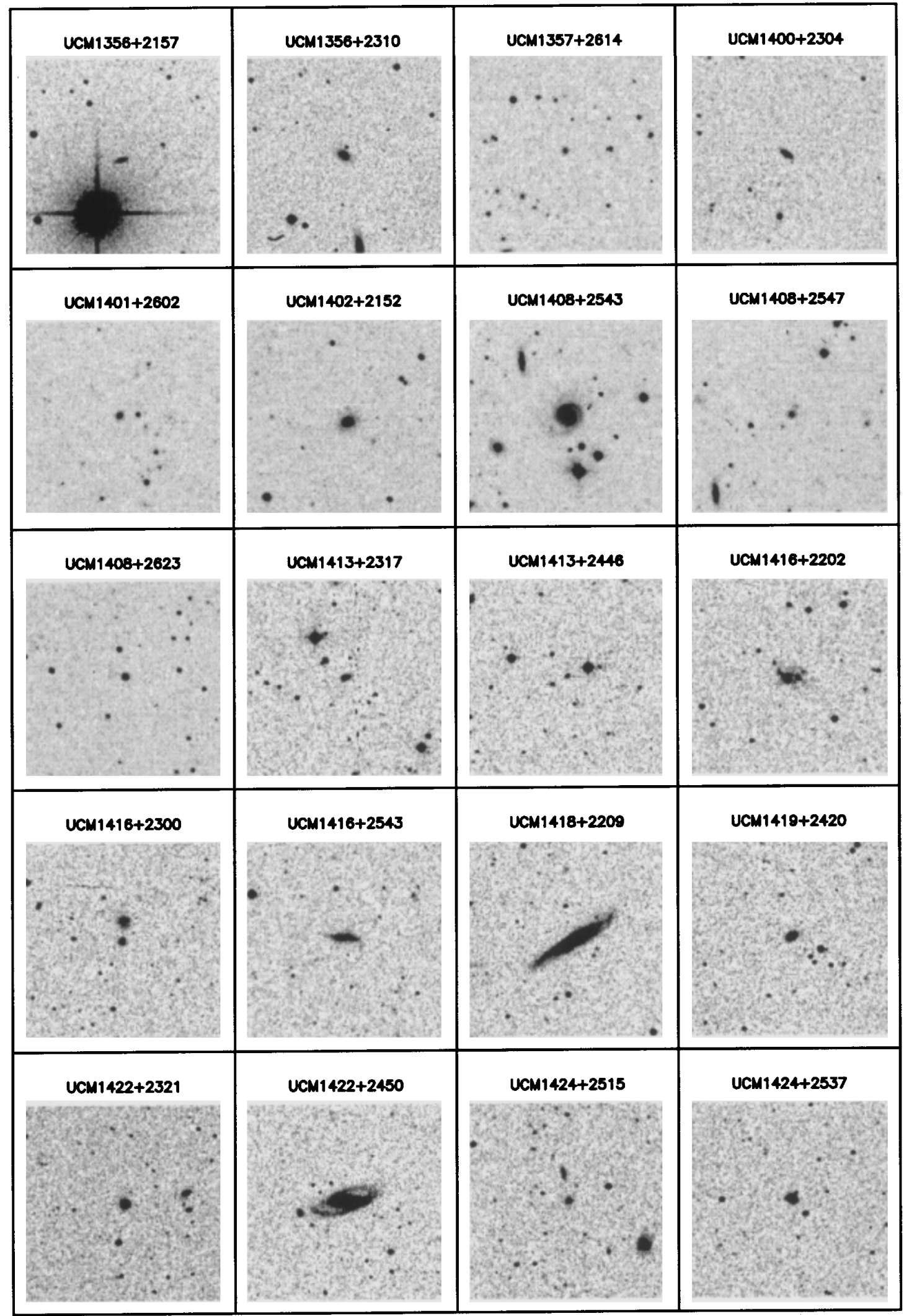

FIG. 12.-Continued

\& Miyuachi-Isobe 1993). If we restrict our analysis to the common region $\left(13^{\mathrm{h}} 45^{\mathrm{m}}-14^{\mathrm{h}} 30^{\mathrm{m}}\right)$, the KUG survey has found 94 objects and UCM 26 candidates; only seven have been selected by both surveys. Thus, only $27 \%$ of UCM galaxies are also KUG objects. Since the KUG survey selects objects by their colors, it is not redshift limited. We expect that a fraction of the KUGs have a redshift that prevents detection by us. For comparison, Comte et al. 


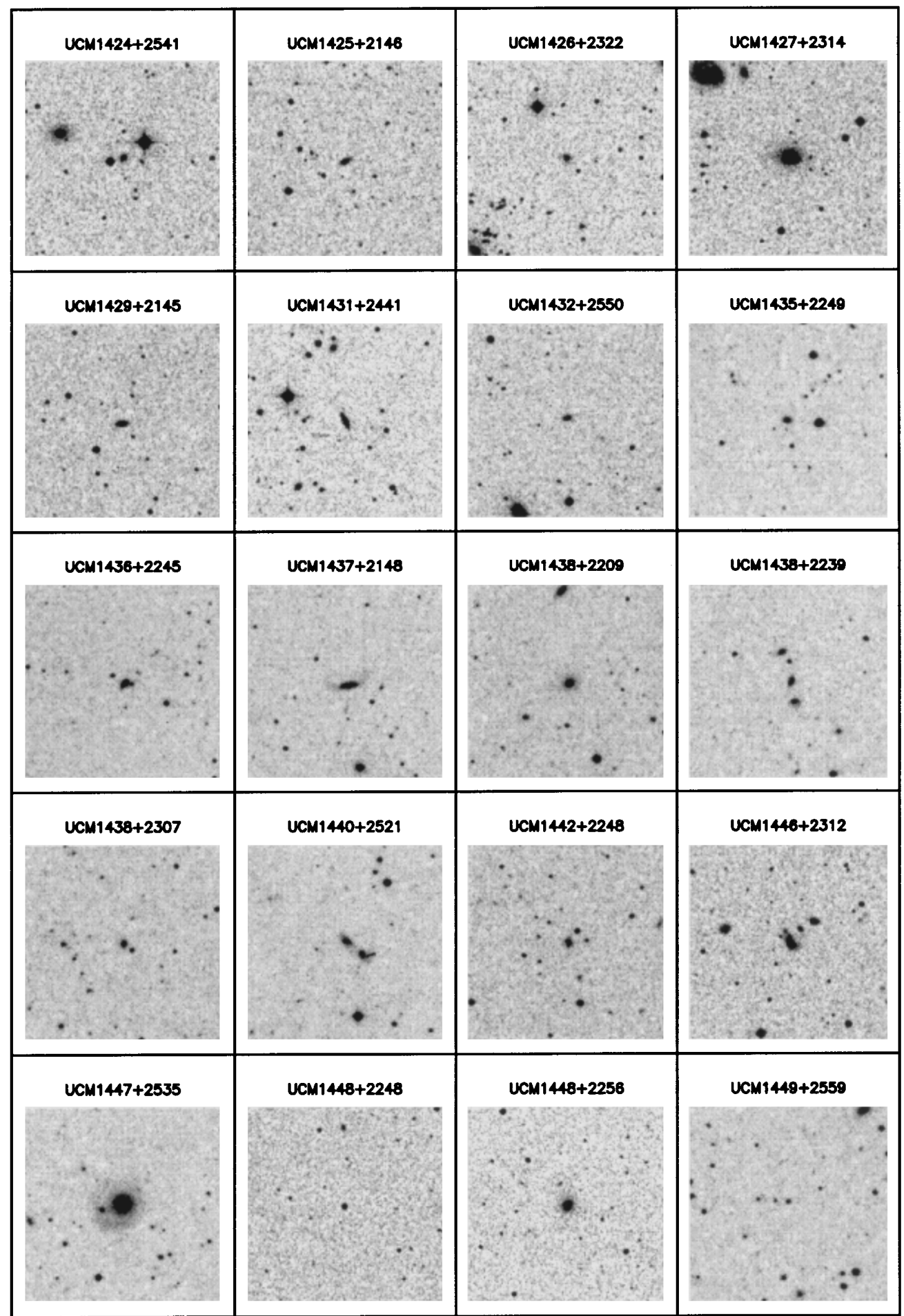

FIG. 12.-Continued

(1994) found $25 \%$ of KUGs with $z>0.04$. The number density of KUGs is 1.8 objects per $\mathrm{deg}^{2}$ for the total survey, outnumbering the surface density of UCM by a factor of 3 .

There are five galaxies of the Wasilewski (1983) catalog of emission-line galaxies that have also been detected by our survey. Although the detection technique is similar (objective-prism), he used a IIIaJ emulsion to register $\mathrm{H} \beta$ and $[\mathrm{O} \mathrm{III}]$ lines. The one galaxy undetected by us is WAS $82\left(m_{B}=15.5\right)$, due to a saturation problem (see $\left.\S 6\right)$. 


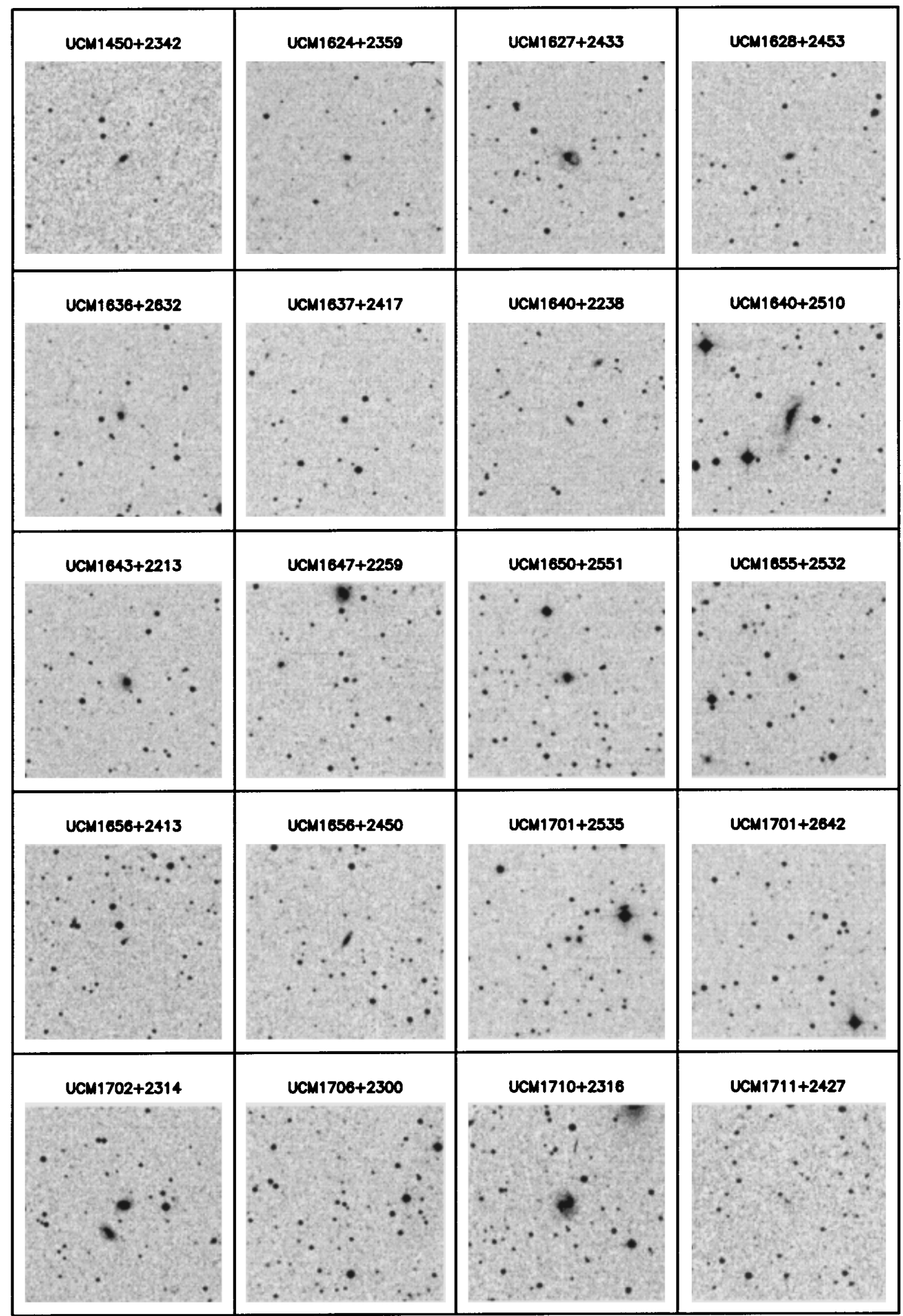

FIG. 12.-Continued

\section{SUMMARY}

We have presented the UCM survey List 3. This new research is the scientific continuation of the previous two lists, but in List 3 we have applied an automatic procedure developed and tested by us in a previous work in order to improve the results obtained up to now using a visual search for candidates. List 3 covers $189 \mathrm{deg}^{2}$ in nine fields, and yields a total number of 113 candidate $\mathrm{H} \alpha$ emission-line galaxies, giving an overall density of 0.59 candidates per $\operatorname{deg}^{2}$. 


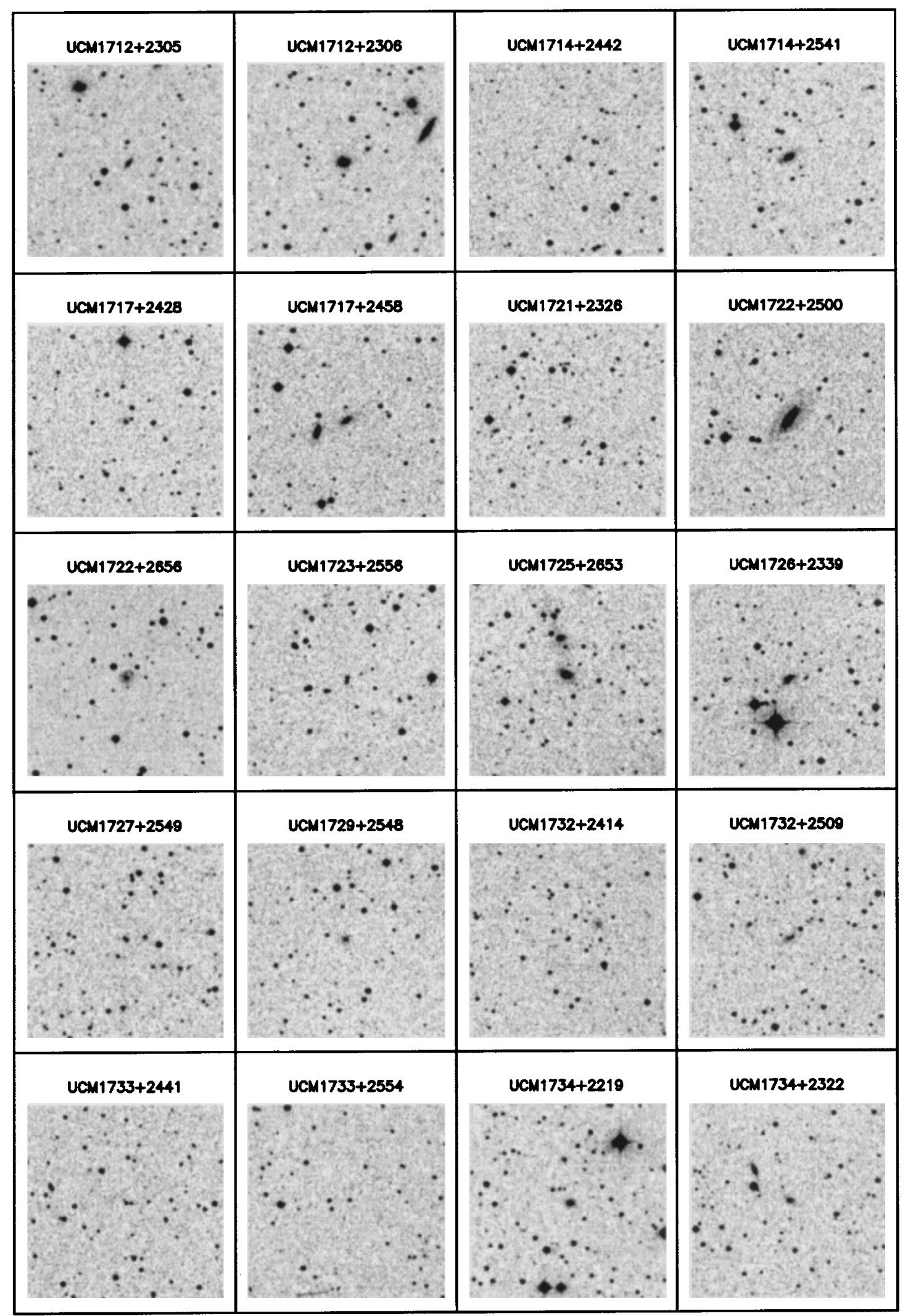

FIG. 12.-Continued

The nine fields were acquired in direct and objectiveprism modes, and the plates were scanned using the MAMA machine. This procedure has permitted us to recover a great amount of information directly from the plates, allowing us to perform statistical analysis of the sample before carrying out photometric or spectroscopic observations. We have derived high-precision coordinates, magnitudes in the blue band, sizes, and redshifts for the whole sample of candidates, all with moderate and known errors. Because we work with uncalibrated plates, all these 


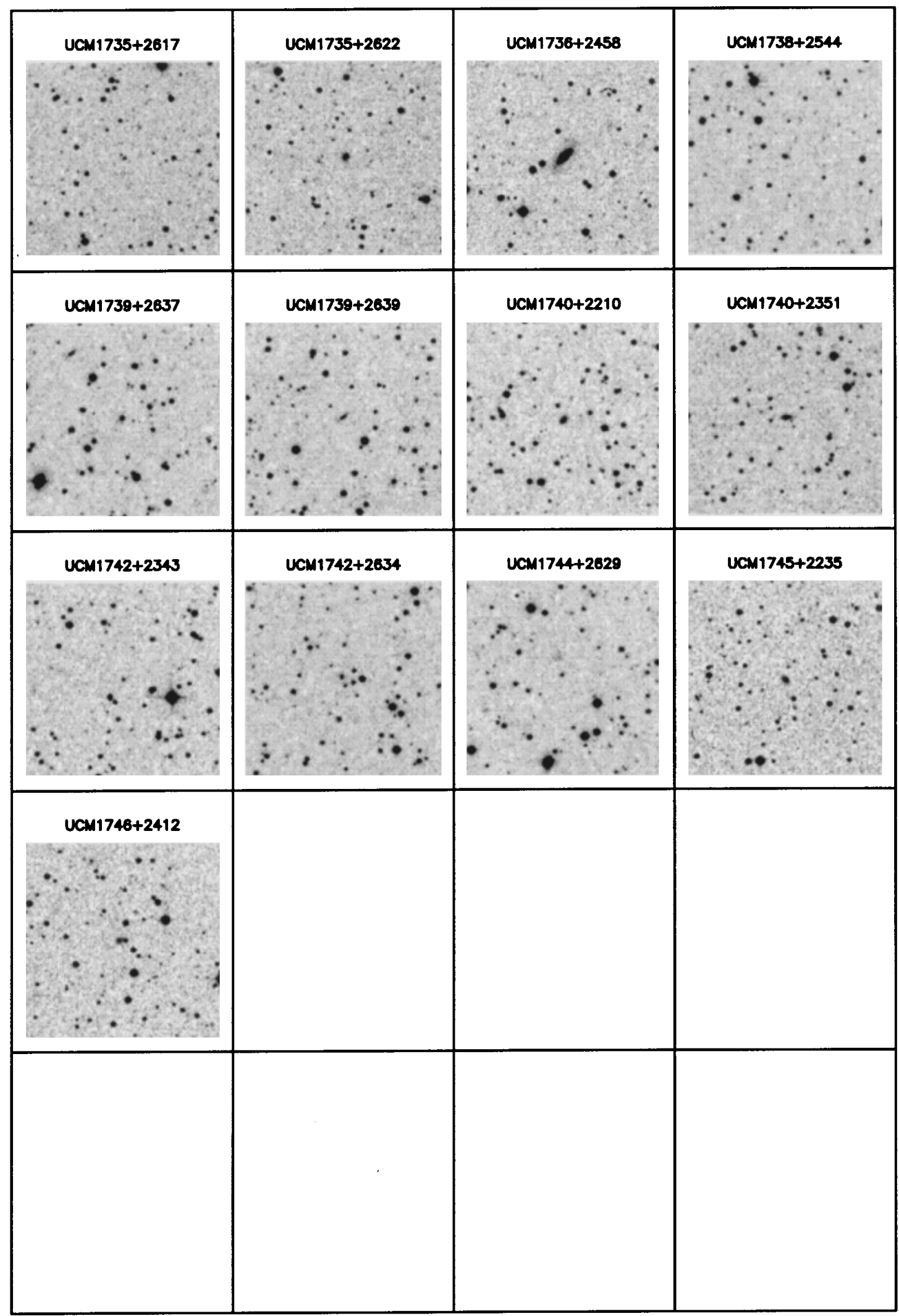

FIG. 12.-Continued

parameters are obtained by comparing our photographic data with several parameters for objects recovered from various databases. These external calibrations allow for the determination of the parameters noted above.
The determination of the dispersion curve of the prism used in the survey has permitted us to estimate the redshift for the whole sample of candidates by measuring the position of the $\mathrm{H} \alpha$ line in the prism spectrum in relation to the 
location of a constant reference point obtained using astrometric calibration. The $1 \sigma$ error in the computation of the redshift by this method is $0.004\left(1200 \mathrm{~km} \mathrm{~s}^{-1}\right)$. A comparison with known objects shows the precision of the method developed.

The photometric data obtained for the sample (apparent and absolute magnitudes and sizes) follow the behavior derived for the two previous lists using specific photometric observations. This result suggests that the automatic procedure selects a sample of galaxies that is comparable with those obtained in the previous lists.

The success of the new automatic procedure adopted in this third list is marked by both the establishment of objective criteria for the selection of candidates and the extraction of several quantitative parameters, in comparison with the lack of such information in previous, visual-based lists.

We would like to gratefully acknowledge the inestimable observation support received from the Calar Alto Observa- tory staff, specially from Kurt Birkle. We also express our thanks to Jean Guibert and the MAMA staff for their friendly assistance and warm hospitality. We would like to thank Armando Gil de Paz, Javier Cenarro, and Nicolás Cardiel for their helpful comments and valuable suggestions. We would also like to thank Marion Schmitz from the NED catalog and Heinz Andernach from Guanajuato University for their help in the revision of Table 2, and the anonymous referee for his useful suggestions and comments that improved this paper. This work has made use of the NASA/IPAC Extragalactic Database (NED), which is operated by the Jet Propulsion Laboratory, Caltech, under contract to the National Aeronautics and Space Administration. This research was supported in part by the Spanish Programa Sectorial de Promoción General del Conocimiento under grants PB96-0645, and PB96-0610.
Alonso, O. 1996, Ph.D. thesis, Univ. Complutense de Madrid

Alonso, O., Zamorano, J., Rego, M., \& Gallego, J. 1995, A\&AS, 113, 399

Alonso-Herrero, A., Aragón-Salamanca, A., Zamorano, J., \& Rego, M. 1996, MNRAS, 278, 417

Berger, J., Cordoni, J. P., Fringant, A. M., Guibert, J., Moreau, O., Reboul, H., \& Vanderriest, C. 1991, A\&AS, 87, 389

Birkle, K. 1984, in IAU Colloq. 78, Astronomy with Schmidt-Type Telescopes, ed. M. Capaccioli (Dordrecht: Reidel), 203

Comte, G., Augarde, R., Chalabaev, A., Kunth, D., \& Maehara, H. 1994, A\&A, 285, 1

de Vaucouleurs, G. 1984, in IAU Colloq. 78, Astronomy with SchmidtType Telescopes, ed M. Capaccioli (Dordrecht: Reidel), 409

Fisher, K. B., Huchra, J. P., Strauss, M. A., Davis, M., Yahil, A., \& Schlegel, D. 1995, ApJS, 100, 69

Fukugita, M., Shimasaku, K., \& Ichikawa, T. 1995, PASP, 107, 945

Gallego, J., Zamorano, J., Aragón-Salamanca, A., \& Rego, M. 1995, ApJ, $455, \mathrm{~L} 1$

Gallego, J., Zamorano, J., Rego, M., Alonso, O., \& Vitores, A. G. 1996, A\&AS, 120, 323

Gallego, J., Zamorano, J., Rego, M., \& Vitores A. G. 1997, ApJ, 475, 502

Gil de Paz, A., Aragón-Salamanca, A., Zamorano, J., Gallego, J., \& Alonso-Herrero, A. 1998, in preparation

Guibert, J., \& Moreau, O. 1991, Messenger, 64, 69

Huchra, J. P., Geller, M. J., \& Corwin, H. G. J. 1995, ApJS, 99, 391

Kinman, T. D. 1984, in IAU Colloq. 78, Astronomy with Schmidt-Type

Telescopes, ed. M. Capaccioli (Dordrecht: Reidel), 409

\section{REFERENCES}

Kroll, P., \& Neugebauer, P. 1993, A\&A, 273, 341

MacAlpine, G. M., Smith, S. B., \& Lewis, D. W. 1977, ApJS, 34, 95

Mohan, V., \& Crézé, M. 1987, A\&A, 68, 529

Moreau, O. 1992, Ph.D. thesis, Univ. Paris

Moss, C., Whittle, M., \& Irwin, M. J. 1988, MNRAS, 232, 381

Paturel, G., Fouqué, P., Bottinelli, L., \& Gouguenheim, L. 1989, A\&A, 80, 299

Paturel, G., Fouqué, P., Lauberts, A., Valentijn, E. A., Corwin, H. G., \& de Vaucouleurs, G. 1987, A\&A, 184, 86

Reid, N., \& Gilmore, G. 1982, MNRAS, 201, 73

Roeser, S., \& Bastian, U. 1991, PPM Star Catalogue (Heidelberg: Spektrum)

Surace, C., \& Comte, G. 1994, A\&A, 281, 653

Takase, B., \& Miyauchi-Isobe, N. 1993, Publ. Natl. Astron. Obs. Japan, 3, 169

Thuan, T. X., \& Martin, G. E. 1981, ApJ, 247, 823

Vitores, A. G., Zamorano, J., Rego, M., Alonso, O., \& Gallego, J. 1996a, A\&AS, 118,7

Vitores, A. G., Zamorano, J., Rego, M., Gallego, J., \& Alonso, O. 1996b, A\&AS, 120, 385

Wasilewski, A. J. 1983, ApJ, 272, 68

Zamorano, J., Rego, M., Gallego, J., Vitores, A. G., González-Riestra, R., \& Rodríguez-Caderot, G. 1994, ApJS, 95, 387 (List 1)

Zamorano, J., Gallego, J., Rego, M., Vitores, A. G., \& Alonso, O. 1996, ApJS, 105, 343 (List 2) 